\title{
Spin chain overlaps and the twisted Yangian
}

\section{Marius de Leeuw, ${ }^{a}$ Tamás Gombor, ${ }^{b}$ Charlotte Kristjansen, ${ }^{c}$ Georgios Linardopoulos ${ }^{d}$ and Balázs Pozsgay ${ }^{e}$}

\author{
${ }^{a}$ School of Mathematics and Hamilton Mathematics Institute, \\ 17 Westland Row, Trinity College Dublin, Dublin 2, Ireland \\ ${ }^{b}$ Lendület Holographic QFT Group, Wigner Research Centre for Physics, \\ Konkoly-Thege Miklós u. 29-33, H-1121 Budapest, Hungary \\ ${ }^{c}$ Niels Bohr Institute, University of Copenhagen, \\ Blegdamsvej 17, DK-2100 Copenhagen Ø, Denmark \\ ${ }^{d}$ Institute of Nuclear and Particle Physics, N.C.S.R., "Demokritos", \\ 15310 Agia Paraskevi, Greece \\ ${ }^{e}$ MTA-BME Quantum Dynamics and Correlations Research Group, \\ Department of Theoretical Physics, Budapest University of Technology and Economics, Budapest, \\ Budafoki út 8, 1111 Budapest, Hungary \\ E-mail: mdeleeuw@maths.tcd.ie, gombor.tamas@wigner.mta.hu, \\ kristjan@nbi.dk, glinard@inp.demokritos.gr, pozsi@eik.bme.hu
}

ABSTRACT: Using considerations based on the thermodynamical Bethe ansatz as well as the representation theory of twisted Yangians we derive an exact expression for the overlaps between the Bethe eigenstates of the $\mathrm{SO}(6)$ spin chain and matrix product states built from matrices whose commutators generate an irreducible representation of $\mathfrak{s o}(5)$. The latter play the role of boundary states in a domain wall version of $\mathcal{N}=4$ SYM theory which has non-vanishing, $\mathrm{SO}(5)$ symmetric vacuum expectation values on one side of a codimension 1 wall. This theory, which constitutes a defect CFT, is known to be dual to a D3-D7 probe brane system. We likewise show that the same methodology makes it possible to prove an overlap formula, earlier presented without proof, which is of relevance for the similar D3-D5 probe brane system.

KeYwords: Boundary Quantum Field Theory, Integrable Field Theories, Supersymmetric Gauge Theory, AdS-CFT Correspondence

ARXIV EPRINT: 1912.09338 


\section{Contents}

1 Introduction and summary 1

2 One-point functions in AdS/dCFT 2

2.1 The $\mathrm{SO}(5)$ symmetric domain wall theory 2

2.2 One-point functions from matrix product states 3

2.3 Exact results for one-point functions 5

2.4 Strategy of derivation 6

$\begin{array}{lll}3 & \text { Integrability tools for overlaps } & 7\end{array}$

$\begin{array}{lll}3.1 & \text { The integrable boundary reflection matrix } & 7\end{array}$

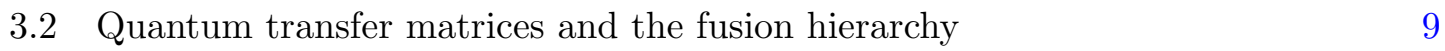

$\begin{array}{lll}3.3 & \text { TBA and overlaps } & 12\end{array}$

4 Application of the TBA $\quad \mathbf{1 5}$

$\begin{array}{lll}4.1 & \text { Overlaps with symmetry class }(\mathrm{SU}(3), \mathrm{SO}(3)) & 15\end{array}$

$\begin{array}{lll}4.2 & \text { An overlap with symmetry class }(\mathrm{SO}(6), \mathrm{SO}(5)) & 17\end{array}$

$\begin{array}{lll}4.3 & \text { An overlap with symmetry class }(\mathrm{SO}(6), \mathrm{SO}(3) \times \mathrm{SO}(3)) & 18\end{array}$

5 The twisted Yangian $\quad 19$

$\begin{array}{llr}5.1 \text { Approach } & 19\end{array}$

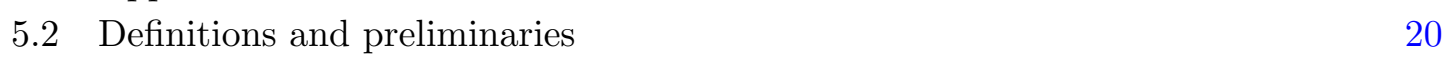

5.2.1 Lie algebras and highest weight representations 20

$\begin{array}{lll}5.2 .2 & \text { Matrix operators } & 21\end{array}$

5.2.3 Yangians and highest weight representations 21

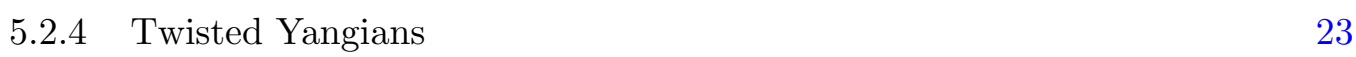

$5.3 \quad(\mathrm{SU}(3), \mathrm{SO}(3))$ case $\quad 26$

5.3.1 Odd $k=2 s+1 \quad 26$

$\begin{array}{ll}\text { 5.3.2 Even } k=2 s+1 & 29\end{array}$

$5.4 \quad(\mathrm{SO}(6), \mathrm{SO}(5))$ case $\quad 30$

6 Conclusion and outlook 32

A Limiting formulas for the overlaps $\quad 33$

B Calculations with the twisted Yangian $\boldsymbol{Y}^{+}(\mathbf{3}) \quad \mathbf{3 4}$

B.1 Odd $k=2 s+1 \quad 34$

B.2 Even $k=2 s+1 \quad 37$

C Calculations with the twisted Yangian $X\left(\mathfrak{s o}_{6}, \mathfrak{s o}_{5}\right) \quad 40$ 


\section{Introduction and summary}

A surprisingly fruitful cross-fertilization between holography and statistical physics has taken place in recent years due to a common interest in overlaps between Bethe eigenstates of integrable systems and states which are not easily expressible in terms of eigenstates. In statistical physics the latter type of state typically constitutes the initial state in a quantum quench of an integrable system and the overlaps are key to investigating the time development after the quench, see f.inst. [1-8]. In holography the typical state of interest is a so-called matrix product state which encodes information about the vacuum of the holographic system and the overlaps give the expectation value of the theory's operators in the vacuum state [9-14].

From the point of view of holography, systems which are amenable to an analysis of this type are domain wall versions of $\mathcal{N}=4 \mathrm{SYM}$ theory where the vacuum is different on the two sides of a codimension 1 wall. More precisely, the vacua on the two sides of the wall differ by some of the scalar fields taking nonzero vacuum expectation values (vevs) on one side, say for $x_{3}>0$. These field theories constitute defect conformal field theories (dCFTs) and are dual to probe brane systems with configurations of background gauge fields which lead to nontrivial flux or instanton number [15-21]. There are essentially three such systems, one being a D3-D5 probe brane system and the two others being D3-D7 probe brane systems, cf. table 1. In dCFT's one can encounter non-vanishing one-point functions and due to the nontrivial vacuum expectation values this will happen already at tree-level for the setups in question. As first pointed out in $[9,10]$ the tree level onepoint functions of scalar operators for $x_{3}>0$ can conveniently be expressed as an overlap between a matrix product state and a Bethe eigenstate of the $\mathrm{SO}(6)$ integrable spin chain.

Both from the point of view of statistical physics and from the point of view of holography it is interesting to understand when a certain initial state or a matrix product state is "solvable", i.e. under what circumstances the various physical quantities associated to the state can be computed exactly. One class of such quantities are the overlaps with the Bethe eigenstates of the system. Based on the experience from a number of concrete studies of overlaps [9-12, 22-25] a proposal for an integrability criterion was put forward in [5]. An initial state or matrix product state was said to be integrable if it was annihilated by all the conserved charges of the integrable system, which were odd under space-time parity.

All the overlaps which were known in closed form at that time were compatible with this definition of integrability. This was in particular true for the dCFT dual to the $1 / 2$ supersymmetric D3-D5 probe brane system with background gauge field flux where a closed expression for all the one-point functions of the scalar sector had been found [13]. When applied to the two non-supersymmetric D3-D7 probe brane setups with flux the proposed integrability criterion implied a characterization of one of them as integrable and the other one as non-integrable cf. table 1. This led to an apparent puzzle since for none of them a closed expression for the overlaps had been found [12, 14].

In the present paper we resolve this apparent puzzle and provide another strong consistency check of the proposed integrability criterion by explicitly deriving a closed formula for the overlap giving the scalar one-point functions of the $\mathrm{SO}(5)$ symmetric D3-D7 probe 


\begin{tabular}{|c|c|c|c|}
\hline & D3-D5 & D3-D7 & D3-D7 \\
\hline Supersymmetry & $1 / 2$-BPS & None & None \\
\hline Brane geometry & $\mathrm{AdS}_{4} \times \mathrm{S}^{2}$ & $\mathrm{AdS}_{4} \times \mathrm{S}^{2} \times \mathrm{S}^{2}$ & $\mathrm{AdS}_{4} \times \mathrm{S}^{4}$ \\
\hline $\mid$ MPS $\rangle$ & Integrable & Non-integrable & Integrable \\
\hline Closed overlap formula & Yes & No & Yes (this work) \\
\hline
\end{tabular}

Table 1. The dCFT versions of $\mathcal{N}=4$ SYM theory with non-vanishing vevs and their dual string theory configurations. The discussion of the integrability properties of the corresponding matrix product states can be found in $[13,14]$ and the closed expression for the overlap formula for the D3-D5 case appears in [9-11, 13] for tree level and in [26] for one-loop, see also [27, 28].

brane setup with non-vanishing instanton number. Our proof combines analyticity considerations related to the thermodynamical Bethe ansatz with representation theory of twisted Yangians. We also show that a similar approach can be used to prove the overlap formula for the D3-D5 probe brane system, earlier presented without proof. For simplicity we carry out the proof only for an $\mathrm{SU}(3)$ subsector in this case.

We start in section 2 by briefly discussing the dCFT with $\mathrm{SO}(5)$ symmetric vevs and the matrix product state which is used to calculate its one-point functions. In this section we also present the closed form of the overlap for any scalar operator and for any value of the instanton number. Subsequently, in section 3, we introduce some integrability tools that will play an important role for our analysis. In particular, we explain the connection between the overlaps and the Y-system. Based on analyticity considerations for the $Y$-system we then in section 4 derive overlap formulas for a set of simple "base" states with respectively $\mathrm{SO}(3)$ and $\mathrm{SO}(5)$ symmetry. In section 5 we review elements of the representation theory of twisted Yangians and use these ideas to relate the base states to the desired matrix product states and in that way derive the desired overlap formulas. Section 6 contains our conclusion and outlook. Some technical details are relegated to appendices.

\section{One-point functions in AdS/dCFT}

\subsection{The SO(5) symmetric domain wall theory}

We will be considering a domain wall version of $\mathcal{N}=4$ SYM theory with gauge group $\mathrm{U}(N)$ where the theory has a nontrivial vacuum on one side of the wall. More precisely, we consider a codimension 1 wall placed at $x_{3}=0$ and we allow for (some of) the scalar fields of the theory to have non-vanishing classical values for $x_{3}>0$. Assuming $\psi^{\mathrm{cl}}=A_{\mu}^{\mathrm{cl}}=0$, the classical values for the scalar fields have to fulfil the equation

$$
\nabla^{2} \phi_{i}^{\mathrm{cl}}=\left[\phi_{j}^{\mathrm{cl}},\left[\phi_{j}^{\mathrm{cl}}, \phi_{i}^{\mathrm{cl}}\right]\right], \quad i=1, \ldots, 6 .
$$

By allowing the classical fields to depend on the distance from the defect $x_{3}$ one can obtain a defect CFT. An $\mathfrak{s o ( 5 ) ~ s y m m e t r i c ~ s o l u t i o n ~ w i t h ~ s u c h ~ s p a c e - t i m e ~ d e p e n d e n c e ~ w a s ~ f o u n d ~}$ 
in $[16,29]$

$$
\phi_{i}^{\mathrm{cl}}(x)=\frac{1}{\sqrt{2} x_{3}}\left(\begin{array}{cc}
G_{i} & 0 \\
0 & 0
\end{array}\right), \quad i=1, \ldots, 5, \quad \phi_{6}^{\mathrm{cl}}(x)=0, \quad x_{3}>0,
$$

where the classical fields are $N \times N$ matrices containing the sub-matrices $G_{i}$ of dimension $d_{G} \times d_{G}$ with

$$
d_{G}=\frac{1}{6}(n+1)(n+2)(n+3), \quad n \in \mathbb{Z} .
$$

They can be constructed starting from a four-dimensional representation of the Clifford algebra $\mathfrak{s o}(5)$

$$
\left\{\gamma_{i}, \gamma_{j}\right\}=2 \delta_{i, j} \mathbb{I}_{4 \times 4},
$$

and symmetrizing the $\mathrm{n}$-fold tensor product ${ }^{1}$

$$
G_{i}=\frac{1}{2}(\underbrace{\gamma_{i} \otimes 1 \otimes \cdots \otimes 1}_{n \text { factors }}+\cdots+1 \otimes \cdots \otimes 1 \otimes \gamma_{i})_{\mathrm{sym}} .
$$

The commutators of $G_{i}$ generate a $d_{G}$-dimensional irreducible representation of $\mathfrak{s o}(5)$. We refer to section 5 for a discussion of further properties of the $G_{i}$. For $x_{3}<0$ all fields are considered to be of dimension $\left(N-d_{G}\right) \times\left(N-d_{G}\right)$ with vanishing classical values implying that the gauge group of the field theory is different on the two sides of the wall, namely (broken) $\mathrm{U}(N)$ for $x_{3}>0$ and $\mathrm{U}\left(N-d_{G}\right)$ for $x_{3} \leq 0$ respectively. ${ }^{2}$

This domain wall solution of $\mathcal{N}=4$ SYM theory has a string theory dual consisting of a D3-D7 probe brane system where the D7-brane probes have geometry $\mathrm{AdS}_{4} \times \mathrm{S}^{4}$ and a nontrivial instanton bundle on the $\mathrm{S}^{4}$ carries instanton number equal to $d_{G}$. The probe is the string theory analogue of the gauge theory wall and the change in gauge group across the wall is reflected in $d_{G}$ out of the $N$ D3-branes being dissolved into D7-branes as $x_{3} \rightarrow 0+[33]$. The probe brane system is stable in the parameter region

$$
\frac{\lambda}{\pi^{2}(n+1)(n+3)}<\frac{2}{7}
$$

where $\lambda$ is the 't Hooft coupling, proportional to the inverse string tension according to the AdS/CFT dictionary.

\subsection{One-point functions from matrix product states}

Due to the restricted amount of symmetries of defect CFTs these theories allow for additional classes of correlation functions compared to ordinary CFTs and their two and three-point functions are more involved than for ordinary CFTs. We shall normalize our

\footnotetext{
${ }^{1}$ For the implementation of the symmetrization of the tensor product, see for instance [12].

${ }^{2}$ The consistency of this setup is confirmed by perturbative calculations. One finds that almost all field excitations for $x_{3}>0$ which are outside the $\left(N-d_{G}\right) \times\left(N-d_{G}\right)$ block become infinitely heavy as the wall is approached and have propagators with support only in the region $x_{3}>0$ [30]. For a few remaining excitations this is not the case but for these one needs to impose Dirichlet or Neumann boundary conditions at the wall to obtain the gauge symmetry $\mathrm{U}\left(N-d_{G}\right)$ exactly at the wall [31, 32]. The few special excitations can be ignored in the large- $N$ limit.
} 
operators such that the two-point functions take the canonical form of an ordinary CFT far from the defect, i.e.

$$
\lim _{z_{3} \rightarrow \infty}\left\langle\mathcal{O}_{i}(x+z) \mathcal{O}_{j}(y+z)\right\rangle=\frac{\delta_{i j}}{|x-y|^{\Delta_{i}+\Delta_{j}}},
$$

where the $\Delta$ 's are the conformal dimensions of the operators involved. Our main object of interest will be the one-point functions which are restricted to take the following form

$$
\left\langle\mathcal{O}_{\Delta}(x)\right\rangle=\frac{C}{x_{3}^{\Delta}}
$$

Due to the nontrivial vevs, one-point functions of operators built from the five scalars $\phi_{1}, \ldots, \phi_{5}$ will have non-vanishing one-point functions for $x_{3}>0$ already at tree level. As is well-known [34] the good conformal operators containing only scalar fields can be described at the lowest loop level as the eigenstates of an integrable $\mathrm{SO}(6)$ spin chain given by the $R$-matrix

$$
R(u)=u(u+2) \mathbb{I}+(u+2) \mathbb{P}-u \mathbb{K},
$$

where $\mathbb{P}$ is the permutation operator and $\mathbb{K}$ is the trace operator. These eigenstates can in turn be expressed in terms of three sets of Bethe roots $\left(\left\{u_{i}\right\}_{i=1}^{N_{0}},\left\{v_{j}\right\}_{j=1}^{N_{+}},\left\{w_{k}\right\}_{k=1}^{N_{-}}\right)$which fulfil a set of algebraic Bethe equations. The $u_{i}$ 's are the so-called momentum-carrying roots. We shall collectively refer to the Bethe roots as $\mathbf{u}$ and the corresponding eigenstate as $|\mathbf{u}\rangle$. Determining the one-point function of a conformal operator at tree level amounts to inserting the vevs from eq. (2.2) into the Bethe wave function describing the operator, a procedure which can conveniently be formulated as calculating the overlap of the Bethe eigenstate with a matrix product state (MPS) of bond dimension $d_{G}$, i.e. $[9,10]$

$$
C=\left(\frac{8 \pi^{2}}{\lambda}\right)^{\frac{L}{2}} L^{-\frac{1}{2}} C_{n}, \quad C_{n}=\frac{\left\langle\mathbf{u} \mid \mathrm{MPS}_{n}\right\rangle}{\langle\mathbf{u} \mid \mathbf{u}\rangle^{\frac{1}{2}}}
$$

where

$$
\left|\mathrm{MPS}_{\mathrm{n}}\right\rangle=\sum_{\vec{i}} \operatorname{tr}\left[G_{i_{1}} \ldots G_{i_{L}}\right]\left|\phi_{i_{1}} \ldots \phi_{i_{L}}\right\rangle
$$

with $n$ referring to the dimension of the representation for the vevs via eq. (2.3). In practice it is more convenient to work with complex combinations of the scalar fields defined as follows

$$
\begin{array}{lll}
X=\phi_{1}+i \phi_{2}, & Y=\phi_{3}+i \phi_{4}, & Z=\phi_{5}+i \phi_{6}, \\
\bar{X}=\phi_{1}-i \phi_{2}, & \bar{Y}=\phi_{3}-i \phi_{4}, & \bar{Z}=\phi_{5}-i \phi_{6} .
\end{array}
$$

The dictionary between Bethe eigenstates $|\mathbf{u}\rangle$ and operators built from complex fields can be found for instance in [34]. 


\subsection{Exact results for one-point functions}

Exploiting the symmetry properties of the $G$-matrices one can derive the following selection rule that needs to be fulfilled in order for an operator to have a non-vanishing one-point function [12]

$$
\left(L, N_{0}, N_{+}, N_{-}\right)=\left(L, N_{0}, N_{0} / 2, N_{0} / 2\right), \quad N_{0} \text { even, }
$$

where $L$ is the number of fields of the operator. Furthermore, it was shown in [13] that the matrix product state $(2.11)$ is annihilated by all the odd charges of the integrable $\mathrm{SO}(6)$ spin chain and hence obeys the integrability criterion proposed in [5]. The fact that the matrix product state is annihilated by all the odd charges gives rise to a number of constraints on the possible sets of Bethe roots. First, the momentum-carrying roots have to come in pairs with opposite sign of the momenta or rapidities. The same is the case for the other two types of roots if their number $N_{0} / 2$ is even. If $N_{0} / 2$ is odd the paired roots must be supplemented by a single additional root at zero $[12,13]$. In the remaining part of the paper we will show that in accordance with the integrability criterion being fulfilled there does exist a closed formula for the one-point functions in the present case. The formula is expressed in terms of objects well-known from the study of integrable spin chains, namely the Gaudin determinant $[35,36]$ which gives the norm of a Bethe eigenstate and three types of Baxter polynomials corresponding to the three types of Bethe roots,

$$
Q_{0}(a)=\prod_{i=1}^{N_{0}}\left(i a-u_{i}\right), \quad Q_{+}(a)=\prod_{j=1}^{N_{0} / 2}\left(i a-v_{j}\right), \quad Q_{-}(a)=\prod_{k=1}^{N_{0} / 2}\left(i a-w_{k}\right)
$$

The overlap formula reads

$$
\frac{\left\langle\mathbf{u} \mid \mathrm{MPS}_{n}\right\rangle^{2}}{\langle\mathbf{u} \mid \mathbf{u}\rangle}=\Lambda_{n}^{2} \cdot \frac{Q_{0}(0) Q_{0}\left(\frac{1}{2}\right)}{\bar{Q}_{+}(0) \bar{Q}_{+}\left(\frac{1}{2}\right) \bar{Q}_{-}(0) \bar{Q}_{-}\left(\frac{1}{2}\right)} \cdot \frac{\operatorname{det} G_{+}}{\operatorname{det} G_{-}},
$$

where the bar on the $Q$ 's signifies that a Bethe root at zero should be excluded from the Baxter polynomial and where $\operatorname{det} G$ is the determinant of the Gaudin matrix which for Bethe states with the roots paired as above factors as $\operatorname{det} G=\operatorname{det} G_{+} \operatorname{det} G_{-}{ }^{3,4}$

The pre-factor $\Lambda_{n}$ is a specific transfer matrix eigenvalue, which will be explained later. For $n=1$ which corresponds to the $G_{i}$ being the Dirac gamma matrices we find for the pre-factor

$$
\Lambda_{1}=\left(1+(-1)^{L}\right) \frac{Q_{0}(1)}{Q_{0}(0)}+(-1)^{N_{-}} \frac{Q_{-}\left(\frac{3}{2}\right)}{Q_{-}\left(\frac{1}{2}\right)}+(-1)^{L+N_{+}} \frac{Q_{+}\left(\frac{3}{2}\right)}{Q_{+}\left(\frac{1}{2}\right)} .
$$

\footnotetext{
${ }^{3}$ As far as we know the first appearance of a finite volume overlap involving this ratio of Gaudin-like determinants was in [37], where a rather general explanation for the ratio was given by focusing on the density of states. The work [37] treated the excited state $g$-functions in integrable QFT, which are analogous to the finite volume spin chain overlaps. In spin chains the same structure was found independently in [23].

${ }^{4}$ For an illustration of the factorization of the Gaudin determinant in a case with nested Bethe ansatz, see $[11]$.
} 
An expression valid for any $n \in \mathbb{N}$ can likewise be derived and takes the form:

$$
\Lambda_{n}=2^{L} \sum_{q=-\frac{n}{2}}^{\frac{n}{2}} q^{L}\left[\sum_{p=-\frac{n}{2}}^{q} \frac{Q_{0}\left(p-\frac{1}{2}\right)}{Q_{0}\left(q-\frac{1}{2}\right)} \frac{Q_{-}(q) Q_{-}\left(\frac{n}{2}+1\right)}{Q_{-}(p) Q_{-}(p-1)}\right]\left[\sum_{r=q}^{\frac{n}{2}} \frac{Q_{0}\left(r+\frac{1}{2}\right)}{Q_{0}\left(q+\frac{1}{2}\right)} \frac{Q_{+}(q) Q_{+}\left(\frac{n}{2}+1\right)}{Q_{+}(r) Q_{+}(r+1)}\right] .
$$

If $N_{0} / 2$ is even the formula immediately gives the value of $\Lambda_{n}$. If $N_{0} / 2$ is odd and $n$ is even there can be singularities of the type 0/0 coming from the Baxter polynomials $Q_{+}$ and $Q_{-}$being evaluated at zero. In this case the formula (2.18) still holds but should be understood in a limiting sense so that for instance for $n=2$ and $N_{0} / 2$ even we get

$$
\Lambda_{2}=2^{L+1} \cdot\left[\frac{\left(1+(-1)^{L}\right)}{2} \cdot \frac{Q_{0}\left(\frac{3}{2}\right)}{Q_{0}\left(\frac{1}{2}\right)}+\frac{Q_{-}(2)}{Q_{-}(0)}+(-1)^{L} \cdot \frac{Q_{+}(2)}{Q_{+}(0)}\right], \quad N_{0} / 2 \text { even, }
$$

whereas for $n=2$ and $N_{0} / 2$ odd the result reads

$$
\begin{aligned}
\Lambda_{2}=2^{L+1} \cdot\left[\frac{\left(1+(-1)^{L}\right)}{2}\right. & \frac{Q_{0}\left(\frac{3}{2}\right)}{Q_{0}\left(\frac{1}{2}\right)}+\frac{Q_{-}(2)}{\bar{Q}_{-}(0)}\left[\frac{Q_{0}^{\prime}\left(\frac{1}{2}\right)}{Q_{0}\left(\frac{1}{2}\right)}-\frac{Q_{-}^{\prime}(1)}{Q_{-}(1)}\right]+ \\
& \left.+(-1)^{L} \cdot \frac{Q_{+}(2)}{\bar{Q}_{+}(0)}\left[\frac{Q_{0}^{\prime}\left(\frac{1}{2}\right)}{Q_{0}\left(\frac{1}{2}\right)}-\frac{Q_{+}^{\prime}(1)}{Q_{+}(1)}\right]\right], \quad N_{0} / 2 \text { odd. }
\end{aligned}
$$

The generalization of this formula to arbitrary values of $n$ can be found in appendix A.

\subsection{Strategy of derivation}

The overlap formula given by (2.16) and (2.18) has a rather intricate structure with the pre-factor of the determinant term involving a sum over products of $Q$-functions. Most overlap formulas for which an explicit derivation has been possible until now have only a single product as a pre-factor. This holds for the overlaps of the XXZ spin chain between Bethe eigenstates and general two-site product states [38], including the Néel state, the dimer state and the $q$-deformed dimer state [22-24]. It also holds for overlaps of the XXX Heisenberg spin chain with matrix product states which are built from the generators of $\mathfrak{s u}(2)$ in the spin-1/2 representation [9]. An exception is the generalization of the latter overlaps to higher representations for which a recursive strategy for the derivation could be pursued [10].

In cases where the pre-factor in the overlap formula consists of a single product the prefactor can be found by making use of the thermodynamical Bethe ansatz and exploiting certain analyticity properties of the $Y$-functions [38]. For instance, this method makes it possible to prove an overlap formula, first presented in [11], for the $\mathrm{SU}(3)$ spin chain between Bethe eigenstates and certain matrix product states built from Pauli-matrices, as we shall show in section 4.1. However, if the pre-factor is more involved, the method only gives its leading behaviour in the thermodynamical limit.

Currently, there exists a number of more involved overlap formulas which have been presented without proof. One gives the overlap formula of the $\mathrm{SU}(3)$ spin chain with 
matrix product states built from generators of $\mathfrak{s u}(2)$ in higher representations and another one gives the overlap formula for the integrable $\mathrm{SO}(6)$ spin chain with similar matrix product states [13]. As a side-track of the investigations of the present paper we shall prove the former of these two formulas which we characterize in terms of symmetries as the ( $\mathrm{SU}(3), \mathrm{SO}(3)$ ) case. Our main goal, however, is to derive the formula (2.16), for which no proposal existed up to now. This case will correspondingly be denoted as the $(\mathrm{SO}(6), \mathrm{SO}(5))$ case.

Our strategy for deriving the overlap formulas is the following. First we compute the overlap for a simple matrix product state built from one or two-site states using the TBA approach. Subsequently we use the representation theory of twisted Yangians to relate the desired more complicated MPS's to the simple ones, invoking in the process a reflection matrix fulfilling the relevant boundary Yang Baxter equation.

In order to verify intermediate steps in the procedure as well as the final formula we also calculated the desired overlaps numerically. The Bethe states were constructed either by using the appendix E.5 of [39] or by explicitly diagonalizing the Hamiltonian. The corresponding Bethe roots have been obtained with the "Fast Bethe Solver" program [40-42]. Details of the tests performed can be found in appendix A.

\section{$3 \quad$ Integrability tools for overlaps}

In this section we present the main ingredients needed for our derivation of the overlap formula. First, we recall the definition of integrable initial states and explain that this concept is related to the existence of an integrable boundary reflection matrix which can be used to form a double-row transfer matrix. Secondly, we review the construction of the so-called fusion hierarchy of the double-row transfer matrix as well as the associated $Y$-functions. Finally, we explain how the $Y$-functions determine the singularity structure of the overlap formulas via the thermodynamical Bethe ansatz (TBA).

\subsection{The integrable boundary reflection matrix}

We consider local integrable spin chains, where the local Hilbert space on each site is $\mathbb{C}^{N}$ with some $N \geq 2$. The model has an associated fundamental $R$-matrix $R(u) \in \operatorname{End}\left(\mathbb{C}^{N} \otimes \mathbb{C}^{N}\right)$ which enjoys a symmetry with respect to a Lie group $\mathcal{G}$. In the concrete examples we will focus on $\mathcal{G}=\mathrm{SU}(N)$ and $\mathcal{G}=\mathrm{SO}(N)$.

We define the monodromy matrix of a homogeneous spin chain of length $L$ as

$$
T(u)=R_{0 L}(u) \ldots R_{02}(u) R_{01}(u) .
$$

The subscript 0 refers to the auxiliary space $V_{0}$, and in our examples $V_{0} \approx \mathbb{C}^{N}$. The transfer matrix is the trace of the monodromy matrix over the auxiliary space:

$$
t(u)=\operatorname{Tr}_{0} T(u)
$$

\footnotetext{
${ }^{5}$ We would like to thank to C. Marboe and D. Volin for informative discussions and for sharing their code with us.
} 
We also define the space-reflected transfer matrix:

$$
\bar{t}(u)=\Pi t(u) \Pi=\operatorname{Tr}_{0} R_{01}(u) \ldots R_{0 L}(u)
$$

An initial state $|\Psi\rangle$ is said to be integrable if the following condition is fulfilled $[5,8]$

$$
t(u)|\Psi\rangle=\bar{t}(u)|\Psi\rangle
$$

We will be interested in a specific type of initial states, namely matrix product states defined for a spin chain with $N$ possible states at each site by

$$
\left|\Psi_{\omega}\right\rangle=\sum_{j_{1}, \ldots, j_{L}=1}^{N} \operatorname{tr}_{a}\left[\omega_{j_{L}} \ldots \omega_{j_{2}} \omega_{j_{1}}\right]\left|j_{L}, \ldots, j_{2}, j_{1}\right\rangle,
$$

where the matrices $\omega_{j}, j=1, \ldots, N$ act on a further auxiliary space $V_{a}$. Typically the matrix product state (MPS) is invariant with respect a subgroup $\mathcal{G}^{\prime} \subset \mathcal{G}$. In this case we say that the symmetry class of the problem is $\left(\mathcal{G}, \mathcal{G}^{\prime}\right)$. In the cases encountered so far the two Lie groups are a symmetric pair. In [8] it was found that an MPS is integrable in the sense described above if it can be embedded into the framework of the (twisted) Boundary Yang-Baxter relation. We now describe this connection.

Let us consider a rapidity dependent two-site block $\psi(u) \in \mathbb{C}^{N} \otimes \mathbb{C}^{N} \otimes \operatorname{End}\left(V_{a}\right)$. It is useful to think about $\psi(u)$ as a collection of matrices $\psi_{a b}(u), a, b=1, \ldots, N$ which act on $V_{a}$. As shown in [8], the matrix product state (3.5) is integrable if there exists a solution $\psi(u)$ to the equation

$$
\check{R}_{23}(u)(\omega \otimes \psi(u))=\check{R}_{12}(u)(\psi(u) \otimes \omega),
$$

where $\check{R}(u)=\mathbb{P} R(u)$. Written out more explicitly

$$
\check{R}_{a b}^{d e}(u) \omega_{d} \psi_{e c}(u)=\check{R}_{b c}^{d e}(u) \psi_{a d}(u) \omega_{e}
$$

This relation was dubbed the "square-root relation" because it involves half the steps of the full Boundary Yang-Baxter (BYB) equation, and implies the initial condition (allowing for an overall numerical factor)

$$
\psi_{j k}(0)=\omega_{j} \omega_{k}
$$

It was also argued in [8] that if certain dressed MPS's are completely reducible, then the square root relation is equivalent to the BYB relation. A familiar form of the BYB can be written down if we identify the $K$-matrix as

$$
K(u)=\sum_{a, b} E_{a b} \otimes \psi_{a b}(u),
$$

where $E_{a b}$ are the elementary matrices acting on $V_{0}$. Then the twisted Boundary YangBaxter relation reads:

$$
K_{2}(v) R_{21}^{t}(-u-v) K_{1}(u) R_{12}(u-v)=R_{21}(u-v) K_{1}(u) R_{12}^{t}(-u-v) K_{2}(v),
$$


where $t$ is partial transposition with respect to one of the spaces:

$$
\left(R^{t}(u)\right)_{a b}^{c d}=R_{a d}^{c b}(u)
$$

The $R$-matrix is symmetric with respect to the full transposition, therefore we can take the partial transpose with respect to either space. Note that in the general case $K$ is a matrix composed of linear operators acting on $V_{a}$. In our main case of interest where the matrices $\omega$ are given by (2.2) one can show that the following two-site block gives a solution of the square root relation as well as the twisted boundary Yang-Baxter equation

$$
\begin{aligned}
& \psi_{a b}(u)=2(u+1) G_{a} G_{b}-2 u(u+1)\left[G_{a}, G_{b}\right]-u\left(4 u^{2}+C\right) \delta_{a b}, \\
& \psi_{66}(u)=u(4 u(u+2)-C),
\end{aligned}
$$

where $a, b=1, \ldots, 5$ and $C$ is the quadratic Casimir

$$
C=\sum_{a=1}^{5} G_{i}^{2}=n(n+4)
$$

Via the relation to the reflection matrix (3.9) we thus have an understanding of the integrability of the matrix product state (2.11) in a scattering picture. This particular reflection matrix plays a key role for our derivation of the overlap formula (2.16) and (2.18).

\subsection{Quantum transfer matrices and the fusion hierarchy}

We now reformulate the overlap as a special case of a quantum transfer matrix as described in $[5,6,8,38]$. First, let us define inhomogeneous initial states

$$
\left|\Psi\left(u_{1}, u_{2}, \ldots, u_{L / 2}\right)\right\rangle=\sum_{i_{1}, \ldots, i_{L}=1}^{N} \operatorname{tr}_{a}\left[\psi_{i_{L}, i_{L-1}}\left(u_{L / 2}\right) \ldots \psi_{i_{2}, i_{1}}\left(u_{1}\right)\right]\left|i_{L}, \ldots, i_{1}\right\rangle,
$$

where $\psi(u)$ is a solution to the BYB. Next, let us consider the scalar product of two matrix product states corresponding to two solutions $\psi_{A}(u)$ and $\psi_{B}(u)$, not necessarily coinciding. For that purpose, we define the dual MPS vectors as

$$
\left\langle\Psi\left(u_{1}, u_{2}, \ldots, u_{L / 2}\right)\right|=\sum_{i_{1}, \ldots, i_{L}=1}^{N} \operatorname{tr}_{a}\left[\psi_{i_{L}, i_{L-1}}\left(-u_{L / 2}\right) \ldots \psi_{i_{2}, i_{1}}\left(-u_{1}\right)\right]\left\langle i_{L}, \ldots, i_{1}\right| .
$$

Here the sign difference is important. Next, let us construct the partition functions

$$
\begin{aligned}
& Z_{A B}\left(v_{1}, \ldots, v_{m} \mid u_{1}, \ldots, u_{L / 2}\right)= \\
& \left\langle\Psi_{B}\left(u_{1}, \ldots, u_{L / 2}\right)\left|\prod_{j=1}^{m} t\left(v_{j} \mid u_{1}, \ldots, u_{L / 2}\right)\right| \Psi_{A}\left(u_{1}, \ldots, u_{L / 2}\right)\right\rangle,
\end{aligned}
$$

where

$$
t\left(v \mid u_{1}, \ldots, u_{L / 2}\right)=\operatorname{Tr}_{0} R_{0 L}\left(v-u_{L / 2}\right) R_{0 L-1}\left(v+u_{L / 2}\right) \ldots R_{02}\left(v-u_{1}\right) R_{01}\left(v+u_{1}\right) .
$$


The $Z_{A B}$ are completely symmetric in both the $u$ - and the $v$-parameters [8]. They can be evaluated in the mirror channel by means of certain double-row transfer matrices. We define

$$
\mathcal{T}_{A B}(u)=\operatorname{Tr}\left(M_{A}(u) K_{B}^{t}(-u)\right)
$$

where

$$
M_{A}(u)=T(u) K_{A}(u) T^{t}(-u),
$$

is the "quantum monodromy matrix". The partition function is then computed as [8]

$$
Z_{A B}\left(v_{1}, \ldots, v_{m} \mid u_{1}, \ldots, u_{L / 2}\right)=\operatorname{Tr}\left[\prod_{j=1}^{L / 2} \mathcal{T}_{A B}\left(u_{j} \mid v_{1}, \ldots, v_{m}\right)\right] .
$$

In the physical cases we require that the "initial state" and the "final state" are adjoints of each other.

Let us explain the construction of the fusion hierarchy in the case where the symmetry group is $\mathrm{SU}(N)$ and the physical spaces of the spin chain carry the defining representation. The construction of the fusion hierarchy is rather similar for the periodic case and the boundary case. It is known that picking any representation $\Lambda$ of $\mathrm{SU}(N)$ we can construct a transfer matrix $t^{\Lambda}(u)$ (be it a single-row or a double-row transfer matrix) where the auxiliary space carries the representation $\Lambda$. The representations are indexed by Young diagrams, and a special role is played by the rectangular diagrams. For a Young diagram with $a$ rows and $m$ columns let $t_{m}^{(a)}(u)$ denote the corresponding fused transfer matrix [43]. These transfer matrices satisfy the Hirota equation (T-system)

$$
\begin{aligned}
t_{m}^{(a)}\left(u+\frac{i}{2}\right) t_{m}^{(a)}\left(u-\frac{i}{2}\right) & =t_{m+1}^{(a)}(u) t_{m-1}^{(a)}(u)+t_{m}^{(a-1)}(u) t_{m}^{(a+1)}(u), \\
a & =1, \ldots, N-1, \quad m=1,2, \ldots
\end{aligned}
$$

The Hirota equation has various forms depending on certain "gauge choices." We refer to [43] for the discussion of the various conventions. Picking a common eigenvector of the transfer matrices we define the $Y$-functions as

$$
Y_{m}^{(a)}(u)=\frac{t_{m-1}^{(a)}(u) t_{m+1}^{(a)}(u)}{t_{m}^{(a-1)}(u) t_{m}^{(a+1)}(u)}
$$

where the $t_{m}^{(a)}(u)$ now refer to the eigenvalues of the transfer matrices. It follows from the Hirota equation that the $\mathrm{Y}$-functions satisfy the $Y$-system

$$
Y_{m}^{(a)}\left(u+\frac{i}{2}\right) Y_{m}^{(a)}\left(u-\frac{i}{2}\right)=\frac{\left(1+Y_{m+1}^{(a)}(u)\right)\left(1+Y_{m-1}^{(a)}(u)\right)}{\left(1+1 / Y_{m}^{(a+1)}(u)\right)\left(1+1 / Y_{m}^{(a-1)}(u)\right)},
$$

where we note that the $Y$-functions are gauge independent.

The double-row quantum transfer matrices (QTM) defined above can be embedded in this framework in a straightforward way. In the case of the $\mathrm{SU}(N)$ symmetric chains we identify

$$
\mathcal{T}_{A B}(u)=t_{1}^{(1)}(u),
$$


whereas for the $\mathrm{SO}(6)$ symmetric chain we have

$$
\mathcal{T}_{A B}(u)=t_{1}^{(2)}(u)
$$

due to the fact that the defining representation of $\mathrm{SO}(6)$ can be identified with the first antisymmetric tensor representation of SU(4), i.e. it is indexed by the Young diagram with two rows and one column.

It is our goal to find the $Y$-functions for the simplest possible case where there are no transfer matrices in (3.17) inserted between the initial and the final state. In this situation $\mathcal{T}_{A B}$ reduces to

$$
\mathcal{T}_{A B}(u)=\operatorname{Tr}\left(K_{A}(u) K_{B}^{t}(-u)\right) .
$$

We embed this simple QTM into the fusion hierarchy, which enables us to compute all $t_{m}^{(a)}$ and eventually all $Y_{m}^{(a)}$. This embedding procedure is straightforward, albeit somewhat involved. It can be done in essentially two ways.

One possibility is performing the fusion of the boundary $K$-matrices explicitly. This procedure was carried out in [7] for a scalar case in the $\mathrm{SU}(3)$ symmetric model. One can perform the computations using symbolic manipulation programs. This gives explicit formulas for the anti-symmetrically fused transfer matrices $t_{1}^{(a)}$. From these functions all $t$-functions can be obtained, either by the so-called Bazhanov-Reshetikhin determinant formula [44], or in the first few cases by direct application of the $T$-system. From this we can also compute the $Y$-functions analytically. In practice only the first few of these are needed to fix the overlaps.

The second method involves the explicit diagonalization of the transfer matrices of the form (3.18). A number of cases have been treated in the literature, from which we can extract the necessary ingredients. In our concrete computations only the easy case (3.26) is needed, but for the structure of the TM eigenvalues we need to understand the generic case. Therefore we introduce the so-called "tableau sum", which is a general method for solving the $T$-system. The idea is to express the transfer matrix eigenvalue as a sum over all allowed semi-standard Young tableaux of the given shape. Let us take $N$ functions $z^{(j)}(u)$ where $j=1, \ldots, N$. The $z$-functions will serve as fundamental ingredients for the solution of the $T$-system. Let $\tau_{k l}$ denote the element of a tableau $\tau$ in row $k=1, \ldots, a$ and column $l=1, \ldots, m$ from the top left. Then the formula for the fused eigenvalues is $[43,44]$

$$
t_{m}^{(a)}(u)=\sum_{\tau}\left[\prod_{\substack{k=1, \ldots, a \\ l=1, \ldots, m}} z^{\left(\tau_{k l}\right)}\left(u+i \frac{a-2 k+1}{2}-i \frac{m-2 l+1}{2}\right)\right] .
$$

Here the sum runs over all allowed semi-standard tableaux of size $(a \times m)$ for the given $N$. The rapidity shifts are such that for the geometric center of the diagram we have zero shift, the shifts are symmetric, and they increase to the right and to the top. For the defining 
transfer matrix the eigenvalue is simply:

$$
t_{1}^{(1)}(u)=\sum_{j=1}^{N} z^{(j)}(u)
$$

The tableau sum is equivalent to the so-called Bazhanov-Reshetikhin determinant formula [44].

In a generic situation the $z^{(j)}(u)$ functions can be expressed using certain "kinematical functions" and ratios of certain $Q$-functions. In our case there is no need to introduce these $Q$-functions, because the defining transfer matrix is always given by (3.26), and the eigenstates of these quantum transfer matrices do not involve any Bethe roots. Nevertheless the $z$-functions can be read off from the diagonalization of the double-row transfer matrices within the Algebraic Bethe Ansatz. We will show explicit examples of this.

\subsection{TBA and overlaps}

Here we make the connection between the overlaps and $Y$-functions. Let us consider Bethe eigenstates given by $N_{1}, \ldots, N_{a}$ rapidities for the various possible types, corresponding to the various nesting levels. We assume that the set of rapidities for each type consists of pairs with opposite sign. The integrability condition also allows a single rapidity at zero for non momentum-carrying roots, but in this subsection we discard those cases for simplicity. The TBA argument presented below is insensitive to the presence or absence of vanishing rapidities. Let us assume that the overlaps with the initial state can be factorized as follows

$$
\frac{\left|\left\langle\Psi_{0} \mid \mathbf{u}\right\rangle\right|^{2}}{\langle\mathbf{u} \mid \mathbf{u}\rangle}=C(L) \times \prod_{a=1}^{N-1} \prod_{j=1}^{N_{a} / 2} v^{(a)}\left(u_{j}^{(a)}\right) \times \frac{\operatorname{det} G_{+}}{\operatorname{det} G_{-}},
$$

where we introduced the one-particle overlap functions $v^{(a)}(u)$. Note that here we only have a single product in front of the determinants. The pre-factor $C(L)$ does not depend on the Bethe rapidities, and in the general case it is of the form

$$
C(L)=C_{0} \alpha^{L}, \quad \alpha \in \mathbb{R}^{+} .
$$

In the following we show that the $Y$-system determines the singularity properties of the overlap functions through the TBA equations. The main ideas of this approach were laid out in [38], where the XXZ model was considered. Here we generalize it to the $\operatorname{SU}(N)$ symmetric models. The main idea is rather simple: we consider large volumes $L$ and the evaluation of the spectral sum $^{6}$

$$
\left\langle\Psi_{0} \mid \Psi_{0}\right\rangle=\sum_{\mathbf{u}} \frac{\left|\left\langle\Psi_{0} \mid \mathbf{u}\right\rangle\right|^{2}}{\langle\mathbf{u} \mid \mathbf{u}\rangle}
$$

where it is understood that we sum only over Bethe root configurations with paired rapidities and a given number of roots of each type. In large volumes the sum on the r.h.s.

\footnotetext{
${ }^{6}$ In statistical physics one would typically require that in the thermodynamic limit $\left\langle\Psi_{0} \mid \Psi_{0}\right\rangle=1$ (but the norm of the MPS can still have subleading pieces which scale to zero exponentially fast in the volume). However, the holographic one-point functions are given via the overlaps with the unnormalized matrix product states.
} 
will be dominated by Bethe states with a well-defined root distribution. This can be determined using the Quench Action approach [1], which is basically the Thermodynamic Bethe Ansatz applied to the spectral sum above, such that the thermal Boltzmann weights are replaced by the overlaps. The idea is to transform the summation over all Bethe states into a functional integral over the Bethe root densities, and to derive a generalized free energy functional which involves both the overlap contribution and the Yang-Yang entropy associated to the given root distribution. This free energy functional can then be minimized, yielding a specific Bethe root distribution describing states that dominate the spectral sum. On the saddle point the value of the free energy functional needs to coincide with the value given by the leading contribution to the norm of the initial state. This argument also explains why we need the Gaudin-like matrices in the overlaps: they are responsible for the correct $\mathcal{O}\left(L^{0}\right)$ terms in the generalized free energy [38].

In large volume the Bethe roots form string solutions. For an $m$-string of particle type $a$ the overlap factor is

$$
v_{m}^{(a)}(u)=\prod_{k=1}^{m} v^{(a)}(u+i(m+1-2 k) / 2) .
$$

In the thermodynamical limit, let us denote the root densities for the $m$-strings of particle type $a$ as $\rho_{m}^{(a)}(\lambda)$. The extensive part of the overlap is then expressed as

$$
\log \frac{\left|\left\langle\Psi_{0} \mid \mathbf{u}\right\rangle\right|^{2}}{\langle\mathbf{u} \mid \mathbf{u}\rangle}=-\sum_{a=1}^{N-1} \sum_{m=1}^{\infty} \int d u g_{m}^{(a)}(u) \rho_{m}^{(a)}(u),
$$

where

$$
g_{m}^{(a)}(u)=-\log v_{m}^{(a)}(u) .
$$

The minus signs above follow merely from some conventions in the earlier literature. Let us also introduce the hole densities $\rho_{m, h}^{(a)}$ and the filling fractions

$$
\eta_{m}^{a}=\frac{\rho_{m, h}^{(a)}}{\rho_{m}^{(a)}+\rho_{m, h}^{(a)}} .
$$

By standard steps we can derive the TBA equations $[1,4]$

$$
\log \eta_{m}^{(a)}=d_{m}^{(a)}+s \star\left[\log \left(1+\eta_{m-1}^{(a)}\right)+\log \left(1+\eta_{m+1}^{(a)}\right)-\log \left(1+1 / \eta_{m}^{(a-1)}\right)-\log \left(1+1 / \eta_{m}^{(a+1)}\right)\right],
$$

where

$$
d_{m}^{(a)}=-g_{m}^{(a)}+s \star\left(g_{m-1}^{(a)}+g_{m+1}^{(a)}\right), \text { with } g_{0}^{(a)}=0,
$$

and

$$
s(u)=\frac{\pi}{\cosh (\pi u)} .
$$

The convolution of two functions is defined as

$$
(f \star g)(u)=\int \frac{d v}{2 \pi} f(u-v) g(v) .
$$


We note that even though the Quench Action TBA (3.36) can be derived using standard steps, this form valid for the $\mathrm{SU}(N)$ symmetric model with the overlap (3.29) is a new result of this work. It follows from (3.32) that the source terms can be written alternatively as

$$
d_{m}^{(a)}=-g_{m}^{(a)}+s \star\left(g_{m}^{(a)+}+g_{m}^{(a)-}\right), \text { with } g_{0}^{(a)}=0 .
$$

Here we used the notation

$$
f^{ \pm}(u)=f\left(u \pm \frac{i}{2}\right)
$$

As explained in [38], the factorized overlap formula implies that the $\eta$-functions satisfy the $Y$-system (3.23). This is rather nontrivial: the additional source terms in (3.36) could in principle modify the algebraic relations between the $Y$-functions. It is only due to the special form (3.37)-(3.40) that the $Y$-system remains intact for the $\eta$-functions, and this follows from the factorizability of the overlap. The $\eta$-functions can be identified with the $Y$-functions derived from the fusion of the boundary transfer matrices $[5,38]$ :

$$
\eta_{m}^{(a)} \equiv Y_{m}^{(a)}
$$

This identification is a boundary (or quench) counterpart of the same relation in the standard thermodynamics, see for example [45]. We use this correspondence to derive the overlap functions. The basic idea is to take the exact $Y$-functions derived from the fusion hierarchy, and to substitute them into the TBA in the integral form (3.36). This will give us the overlap functions. Instead of directly evaluating the convolutions we choose a different path. It was argued in [38] that it is enough to focus on the singularity properties of the $Y$-functions. Let us define the combination

$$
h_{m}^{(a)}(u)=Y_{m}^{(a)}(u) v_{m}^{(a)}(u) .
$$

We substitute the r.h.s. of (3.43) into the integral equation (3.36). This leads to the simple condition

$$
\log \left(h_{m}^{(a)}\right)=s \star\left(\log \left(h_{m}^{(a),+}\right)+\log \left(h_{m}^{(a),-}\right)\right),
$$

which is satisfied if the functions $\log \left(h_{m}^{(a)}\right)$ are free of singularities, which again implies that all $h_{m}^{(a)}$ are free of zeroes or poles within the physical strip. The latter statement can be proven using special properties of the convolution kernel $s(u)$. The r.h.s. of (3.44) can be computed in Fourier space, and from (3.38) we get the Fourier components

$$
s(k)=\frac{1}{2 \cosh (k / 2)} .
$$

If the functions $\log \left(h_{m}^{(a)}\right)$ are free of singularities, then the Fourier transform of the shifted functions are equal to the original Fourier components multiplied by $e^{ \pm k / 2}$. This compensates the multiplication with the Fourier components $s(k)$. However, if there are any singular points within the physical strip then the Fourier component of the shifted functions includes additional pieces. Note that singularities precisely at the boundary of the strip $\Im(u)= \pm 1 / 2$ are allowed. These conditions are rather strong, because the functions 
$h_{m}^{(a)}$ eventually involve all poles or zeroes of the one-particle functions $v^{(a)}$, even when they are originally far from the physical strip. Therefore, these conditions completely fix the analytic structure of $v^{(a)}$. Typically the overlap also contains some numerical pre-factors that do not depend on the Bethe roots. For example for the normalization of the $v^{(a)}$ is not fixed by the above computations, and there can be the additional factor $C(L)$ in (3.29). In principle these factors can be computed from the overlap sum rule and by looking at the overlaps with zero particles [38], however it is often easier to fix them by coordinate Bethe Ansatz computations. In the present work we choose this second option.

\section{Application of the TBA}

In this section we apply the general results of the previous section to determine the overlaps for a set of simple "base" states which will be our starting point for the derivation of overlap formulas for more involved matrix product states encoding information about one-point functions for the D3-D7 as well as the D3-D5 probe brane setup. In section 4.1 we consider the $\mathrm{SU}(3)$ symmetric spin chain and in section 4.2 the $\mathrm{SO}(6)$ symmetric one.

\subsection{Overlaps with symmetry class $(\mathrm{SU}(3), \mathrm{SO}(3))$}

The $Y$-system for the $\mathrm{SU}(3)$ spin chain is given in eqs. (3.23) with $a=1,2$. In the following we will replace these indices with the indices $a=0,+$ so that the index 0 is associated with momentum-carrying Bethe roots and the index + with auxiliary Bethe roots.

The scalar state. Let us define the following "delta-state"

$$
\left|\Psi_{\delta}\right\rangle=\otimes_{j=1}^{L / 2}(|11\rangle+|22\rangle+|33\rangle) .
$$

This state corresponds to the two-site block

$$
\psi_{a b}(u)=\delta_{a b},
$$

which is a constant solution to the BYB. Overlaps and quantum quenches for this state were considered in $[6,7]$ where it was found that the overlap is of the form (3.29) with $C(L)=1$ and

$$
v^{(0)}(u)=v^{(+)}(u)=\frac{u^{2}}{u^{2}+1 / 4} .
$$

Furthermore, it was derived that the first $Y$-functions are

$$
Y_{1}^{(0)}(u)=Y_{1}^{(+)}(u)=\frac{3+8 u^{2}}{4 u^{2}} .
$$

Now we check that the functions $h_{m}^{(a)}(u)$ defined in (3.43) satisfy our requirements. First of all, we can see immediately that $h_{1}^{(0)}$ and $h_{1}^{(+)}$are indeed free of singularities within the physical strip. Going further, we can compute the higher $Y$-functions from the $Y$-system (3.23). In the next cases we get:

$$
h_{1}^{(0)}=h_{1}^{(+)}=\frac{5\left(4 u^{2}+1\right)\left(8 u^{2}+11\right)}{4\left(u^{2}+1\right)\left(8 u^{2}+3\right)} .
$$


Once more we see that the requirement is satisfied. At present we don't have a proof that the requirement will be satisfied for all higher $Y$ functions, but direct computation of the next few cases confirms this. The $Y$-functions are such that $Y_{m}^{(a)}$ have zeroes at $u=0$ if $m$ is even and poles if $m$ is odd. This is consistent with the overlap functions above. However, at present we do not have a proof showing that there are no additional singularities of $\log \left(Y_{m}^{(a)}\right)$ within the physical strip.

MPS with bond dimension 2. Let us define $\left|\mathrm{MPS}_{2}\right\rangle$ as a matrix product state (3.5) with the $\omega_{a}$ being the Pauli matrices, i.e.

$$
\omega_{a}=\sigma_{a}, \quad a=1,2,3,
$$

with $\left[\sigma_{a}, \sigma_{b}\right]=i \varepsilon_{a b c} \sigma_{c}$. Overlaps with this state were found in [11], and quantum quenches were studied in [4]. Once again the overlap is of the simple form (3.29) with $\alpha=\frac{1}{4}, C_{0}=4$ and

More precisely,

$$
v^{(0)}(u)=v^{(+)}(u)=\frac{u^{2}+1 / 4}{u^{2}} .
$$

$$
\frac{\left\langle\mathrm{MPS}_{2} \mid \mathbf{u}\right\rangle^{2}}{\langle\mathbf{u} \mid \mathbf{u}\rangle}=4^{1-L} \cdot \frac{Q_{0}\left(\frac{1}{2}\right) Q_{+}\left(\frac{1}{2}\right)}{Q_{0}(0) \bar{Q}_{+}(0)} \cdot \frac{\operatorname{det} G_{+}}{\operatorname{det} G_{-}} .
$$

The solution of the BYB corresponding to this state is [8]

$$
\psi_{a b}(u)=\sigma_{a} \sigma_{b}+2 u \delta_{a b}
$$

In this case the quantum transfer matrix is actually a matrix. We found that in the physical strip the dominant eigenvalue is produced by the singlet state and we computed the fusion hierarchy for this eigenstate. The first two $Y$-functions take the form:

$$
Y_{1}^{(0)}=Y_{1}^{(+)}=\frac{u^{2}\left(17+8 u^{2}\right)}{\left(1+u^{2}\right)\left(3+4 u^{2}\right)}
$$

and one observes that the requirements for $h_{1}^{(0)}(u)$ and $h_{1}^{(+)}(u)$ are clearly satisfied. Computing higher $Y$-functions from (3.23) we see a pattern that the $Y_{m}^{(a)}$ have poles at $u=0$ if $m$ is even and zeroes if $m$ is odd. This is consistent with the overlap functions above, and this can be considered as an independent derivation of (4.7).

Higher-dimensional MPS. Further integrable MPS's with the same symmetry were studied in [13], i.e.

$$
\left|\mathrm{MPS}_{2 s+1}\right\rangle=\sum_{j_{1}, \ldots, j_{L}=1}^{3} \operatorname{tr}_{A}\left[S_{j_{L}} \ldots S_{j_{2}} S_{j_{1}}\right]\left|j_{L}, \ldots, j_{2}, j_{1}\right\rangle,
$$

where $S_{a}$ are the Hermitian generators of SU(2) in the spin- $s$ representation with dimension $2 s+1$ which satisfy the commutation relations $\left[S_{a}, S_{b}\right]=i \varepsilon_{a b c} S_{c}$. It was found in [13] that the corresponding overlaps include a sum of pre-factors:

$$
\frac{\left\langle\mathbf{u} \mid \mathrm{MPS}_{2 s+1}\right\rangle^{2}}{\langle\mathbf{u} \mid \mathbf{u}\rangle}=\left(\mathbb{T}_{2 s}(0)\right)^{2} \cdot \frac{Q_{0}(0) Q_{0}\left(\frac{1}{2}\right)}{\bar{Q}_{+}(0) \bar{Q}_{+}\left(\frac{1}{2}\right)} \cdot \frac{\operatorname{det} G_{+}}{\operatorname{det} G_{-}},
$$


where

$$
\mathbb{T}_{2 s}(x)=\sum_{a=-s}^{s}(x+i a)^{L} \frac{Q_{0}\left(-i x+\frac{2 s+1}{2}\right) Q_{+}(-i x+a)}{Q_{0}\left(-i x+\left(a+\frac{1}{2}\right)\right) Q_{0}\left(-i x+\left(a-\frac{1}{2}\right)\right)} .
$$

The corresponding solution to the BYB reads [8]:

$$
\psi_{a b}^{(s)}(u)=\delta_{a b}+u^{-1}\left[S_{a}, S_{b}\right]-u^{-2} S_{a} S_{b} .
$$

In this case the TBA method cannot be applied to derive the overlap, and we need the representation theory of the twisted Yangians which we invoke in section 5 to relate $\left|\mathrm{MPS}_{2 s+1}\right\rangle$ to $\left|\Psi_{\delta}\right\rangle$ when $2 s+1$ is odd and to $\left|\mathrm{MPS}_{2}\right\rangle$ when $2 s+1$ is even. This will allow us to prove the overlap formula of relevance for one-point functions in the $\mathrm{SU}(3)$ sector of the D3-D5 probe brane setup [13].

\subsection{An overlap with symmetry class $(\mathrm{SO}(6), \mathrm{SO}(5))$}

We now turn to the $\mathrm{SO}(6)$ symmetric model for which the $Y$-system is given by (3.23) with $a=1,2,3$. In the following we will replace these indices with $a=0,+,-$ such that the index 0 corresponds to the momentum-carrying Bethe roots and the indices,+- correspond to the auxiliary Bethe roots. The defining transfer matrix of the $\mathrm{SO}(6)$ symmetric model is identified with $t_{1}^{(2)}(u)$ of the fusion hierarchy. Let us consider the scalar one-site state

$$
\left|\Psi_{0}\right\rangle=\otimes_{j=1}^{L}|1\rangle
$$

which in terms of fields of $\mathcal{N}=4$ super Yang-Mills theory takes the form

$$
\left|\Psi_{0}\right\rangle=\operatorname{Tr}(Z+\bar{Z})^{\otimes L} .
$$

This corresponds to the MPS with bond dimension 1 given by

$$
\omega_{a}= \begin{cases}1 & a=1 \\ 0 & 1<a \leq 6 .\end{cases}
$$

Clearly this state enjoys a residual $\mathrm{SO}(5)$ symmetry. It is important that this state is not an eigenstate of the model, but integrable. The integrability is proved by finding a solution to the BYB reproducing this state:

$$
\psi_{a b}(u)=(2 u+2) \omega_{a} \omega_{b}-u \delta_{a b} .
$$

We compute the first $Y$-functions using the fusion procedure and find

$$
\begin{aligned}
Y_{1}^{(+)} & =Y_{1}^{(-)}=\frac{5 u^{2}}{3 u^{2}+2}, \\
Y_{1}^{(0)} & =\frac{5\left(4 u^{2}+1\right)\left(4 u^{2}+5\right)}{16 u^{2}\left(4 u^{2}+9\right)} .
\end{aligned}
$$

We can calculate these $Y$-functions in an alternative way since the algebraic Bethe Ansatz for this boundary condition was already treated in [46]. From subsection 3.3 of that work 
we can identify the $z$-functions for an open spin chain with arbitrary sites and boundary condition given by (4.18). The result reads ${ }^{7}$

$$
z_{1}(u)=z_{2}(u)=\frac{u-i / 2}{u+i / 2}, \quad z_{3}(u)=z_{4}(u)=1 .
$$

Afterwards we can use the tableau sum to obtain the $Y$-functions (4.19). Computing higher $Y$-functions we see the following general pattern:

$$
\begin{aligned}
& Y_{m}^{(+)}=Y_{m}^{(-)}=\frac{\alpha_{1, m} u^{2}}{\alpha_{2, m}+\alpha_{3, m} u^{2}}, \\
& Y_{m}^{(0)}=\frac{\left(\alpha_{4, m}+\alpha_{5, m} u^{2}\right)\left(\alpha_{6, m}+\alpha_{7, m} u^{2}\right)}{u^{2}\left(4 u^{2}+(m+2)^{2}\right)},
\end{aligned}
$$

where $\alpha_{\{1 \ldots 6\}, m}$ are integers such that there are no additional poles and zeroes within the physical strip other than the obvious ones at $u=0$. This suggests the following overlap functions:

$$
\begin{aligned}
& v^{(+)}(u)=v^{(-)}(u)=\frac{1}{u^{2}\left(u^{2}+1 / 4\right)} \\
& v^{(0)}(u)=u^{2}\left(u^{2}+1 / 4\right)
\end{aligned}
$$

These overlap functions were confirmed by coordinate Bethe Ansatz computations. More precisely, the exact overlaps read

$$
\frac{\left\langle\Psi_{0} \mid \mathbf{u}\right\rangle^{2}}{\langle\mathbf{u} \mid \mathbf{u}\rangle}=\frac{Q_{0}(0) Q_{0}\left(\frac{1}{2}\right)}{\bar{Q}_{+}(0) \bar{Q}_{+}\left(\frac{1}{2}\right) \bar{Q}_{-}(0) \bar{Q}_{-}\left(\frac{1}{2}\right)} \frac{\operatorname{det} G_{+}}{\operatorname{det} G_{-}} .
$$

In section 5.4 we will show that we can obtain the general $\mathrm{SO}(5)$ symmetric MPS of eq. (2.11) by acting on the state (4.15) with certain transfer matrices. This then allows us to prove the overlap formula (2.16) and (2.18).

\subsection{An overlap with symmetry class $(\mathrm{SO}(6), \mathrm{SO}(3) \times \mathrm{SO}(3))$}

In the $\mathrm{SO}(6)$ symmetric model we consider the state

$$
\left|\Phi^{\mathfrak{s o}(3)}\right\rangle=(Z Z+X X+Y Y+\overline{Z Z}+\overline{X X}+\overline{Y Y})^{\otimes \frac{L}{2}} .
$$

This is an integrable initial state, which corresponds to a constant solution of the BYB. We computed the corresponding $T$-functions and $Y$-functions. The first $Y$-functions are

$$
\begin{aligned}
Y_{1}^{(+)}(u) & =Y_{1}^{(-)}(u)=\frac{5 u^{2}+2}{3 u^{2}}, \\
Y_{1}^{(0)}(u) & =\frac{20 u^{2}+9}{16 u^{2}} .
\end{aligned}
$$

In the language of $\mathrm{SU}(4)$ this state corresponds to the symmetry class $(\mathrm{SU}(4), \mathrm{SO}(4))$. This is analogous to the case of the delta-state considered in section 4.1. Moreover, it can

\footnotetext{
${ }^{7}$ Note, that in the present case the QTM's do not include any sites, cf. eq. (3.26). Thus the $z$-functions are obtained entirely from the reflection matrices.
} 
be shown that the $Y$-functions are the same as they would be for the delta-state of the $\mathrm{SU}(4)$ symmetric model. It follows that the overlap functions are identical to that of the delta-state, but now the same function describes the overlap factors for 3 different types of rapidities:

$$
v^{(0)}(u)=v^{(+)}(u)=v^{(-)}(u)=\frac{u^{2}}{u^{2}+1 / 4} .
$$

Putting everything together we find the overlap

$$
\frac{\left\langle\Phi^{\mathfrak{s o}(3)} \mid \mathbf{u}\right\rangle^{2}}{\langle\mathbf{u} \mid \mathbf{u}\rangle}=\frac{Q_{0}(0) Q_{+}(0) Q_{-}(0)}{Q_{0}\left(\frac{1}{2}\right) Q_{+}\left(\frac{1}{2}\right) Q_{-}\left(\frac{1}{2}\right)} \frac{\operatorname{det} G_{+}}{\operatorname{det} G_{-}} .
$$

This overlap formula was then checked and verified on spin chains of length up to 8 . We expect that by means of representation theory of twisted Yangians we would be able to exploit this result for a proof of the overlap formula giving the one-point functions of the full scalar $\mathrm{SO}(6)$ sector of the D3-D5 probe brane setup [13]. For simplicity, we consider only the proof for the $\mathrm{SU}(3)$ subsector in this case.

\section{The twisted Yangian}

In this section we will demonstrate how to refine the factorized overlap formulas from the TBA approach by using the representation theory of twisted Yangians. Twisted Yangians are the symmetry algebras that naturally arise when part of the symmetry algebra is broken, for example by integrable boundary conditions.

\subsection{Approach}

In this section we give a brief overview of our approach to the computation of overlap formulas by using twisted Yangians. We will carry out the explicit computations and give the details in the following sections.

Consider an $R$-matrix $R(u)$ which satisfies the Yang-Baxter equation

$$
R_{12}(u-v) R_{13}(u) R_{23}(v)=R_{23}(v) R_{13}(v) R_{12}(u-v) .
$$

Corresponding to this $R$-matrix, there is a quantum group defined by the so-called RTT relations

$$
R_{12}(u-v) T_{1}(u) T_{2}(v)=T_{2}(v) T_{1}(u) R_{12}(u-v)
$$

where we defined

$$
T(u)=\sum_{i, j} e_{i j} \otimes t_{i j}(u)
$$

For rational $R$-matrices, this algebra turns out to be a so-called extended Yangian algebra $Y(\mathfrak{g})$ corresponding to some Lie algebra $\mathfrak{g}$. 
Now suppose we impose some boundary conditions corresponding to some reflection matrix $K$ that breaks part of this Yangian symmetry. In particular, suppose that $K$ preserves some subalgebra $\mathfrak{h}$. Such a system is integrable if $K$ satisfies the (twisted) boundary Yang-Baxter equation cf. (3.10)

$$
K_{2}(v) R_{21}^{t}(-u-v) K_{1}(u) R_{12}(u-v)=R_{21}(u-v) K_{1}(u) R_{12}^{t}(-u-v) K_{2}(v),
$$

where we assume that $R$ is symmetric and denote $R^{t}=R^{t_{1}}=R^{t_{2}}$ as the partial transpose.

From the $K$-matrix we can then define a subalgebra $Y^{t w} \subset Y$ by introducing the so-called S-matrix, cf. (3.18)

$$
S(u) \equiv T(u) K(u) T^{t}(-u)
$$

From the reflection equation and the Yang-Baxter equation it is then straightforward to show that

$$
S_{2}(v) R_{21}^{t}(-u-v) S_{1}(u) R_{12}(u-v)=R_{21}(u-v) S_{1}(u) R_{12}^{t}(-u-v) S_{2}(v) .
$$

This can now be interpreted as the defining relations of a new algebra $Y^{t w}$ which is, up to some identifications due to the symmetry properties of the explicit K-matrix, called a twisted Yangian. We will denote the integrable system corresponding to this setup $(\mathfrak{g}, \mathfrak{h})$.

Clearly, any representation of a twisted Yangian will give rise to an integrable initial state [8]. Similarly, it can be argued that the action of the transfer matrix of the twisted Yangian on an integrable initial state gives rise to a new integrable initial state. We will now apply this fact to the two systems that we consider in the paper, $(\mathrm{SU}(3), \mathrm{SO}(3))$ and $(\mathrm{SO}(6), \mathrm{SO}(5))$. In particular, we will consider the representation theory of both twisted Yangians related to our initial states and find explicit expressions for the eigenvalues of the corresponding transfer matrices. From this, we will be able to derive the complete overlap formula for both setups.

\subsection{Definitions and preliminaries}

\subsubsection{Lie algebras and highest weight representations}

We work with Lie algebras $\mathfrak{g l}_{N}$ and $\mathfrak{s o}_{N}$. Let us denote the generators of $\mathfrak{g l}_{N}$ and $\mathfrak{s o}_{N}$ by $E_{i j}$ and $F_{i j}$. For $\mathfrak{g l}_{N}$ the indices run through the set $\{1, \ldots, N\}$. For $\mathfrak{s o}_{2 n}$ and $\mathfrak{s o}_{2 n+1}$ the indices are $\{-n, \ldots,-1,1, \ldots, n\}$ and $\{-n, \ldots,-1,0,1, \ldots, n\}$.

Lie algebra $\mathfrak{g l}_{N}$. The Lie algebra $\mathfrak{g l}_{N}$ is defined by the relations

$$
\left[E_{i j}, E_{k l}\right]=\delta_{j k} E_{i l}-\delta_{i l} E_{k j} .
$$

Let $L\left(\lambda_{1}, \ldots, \lambda_{N}\right)$ be the highest weight rep of $\mathfrak{g l}_{N}$ with highest weights $\lambda_{1}, \ldots, \lambda_{N}$, i.e. there exists a nonzero vector $v \in L\left(\lambda_{1}, \ldots, \lambda_{N}\right)$ such that $L\left(\lambda_{1}, \ldots, \lambda_{N}\right)$ is generated by $v$ and

$$
\begin{array}{ll}
E_{i j} \cdot v=0, & \text { for all } i<j, \\
E_{i i} \cdot v=\lambda_{i} v, & i=1, \ldots, N .
\end{array}
$$

The $L\left(\lambda_{1}, \ldots, \lambda_{N}\right)$ is finite dimensional iff $\lambda_{i}-\lambda_{i+1} \in \mathbb{N}$ for $i=1, \ldots, N-1$. 
Lie algebra $\mathfrak{s o}_{N}$. The Lie algebra $\mathfrak{s o}_{N}$ is defined by the relations

$$
\begin{aligned}
{\left[F_{i j}, F_{k l}\right] } & =\delta_{j k} F_{i l}-\delta_{i l} F_{k j}+\delta_{j,-l} F_{k,-i}-\delta_{i,-k} F_{-j, l}, \\
F_{-j,-i} & =-F_{i, j} .
\end{aligned}
$$

Let $N=2 n$ and $N=2 n+1$ for even and odd $N$ respectively. For any n-tuple $\left(\lambda_{1}, \ldots, \lambda_{n}\right)$ there exists an irreducible highest weight representation $V\left(\lambda_{1}, \ldots, \lambda_{N}\right)$ of $\mathfrak{s o}_{N}$ which is generated by a vector $w$ for which

$$
\begin{array}{ll}
F_{i j} \cdot w=0, & \text { for } i<j, \\
F_{i i} \cdot w=\lambda_{i} w, & \text { for } i=1, \ldots, n .
\end{array}
$$

The representation $V\left(\lambda_{1}, \ldots, \lambda_{N}\right)$ is finite dimensional if and only if

$$
\begin{aligned}
\lambda_{i}-\lambda_{i+1} \in \mathbb{N}, & \text { for } i=1, \ldots, n, \\
-\lambda_{1}-\lambda_{2} \in \mathbb{N}, & \text { if } N=2 n, \\
-2 \lambda_{1} \in \mathbb{N}, & \text { if } N=2 n+1 .
\end{aligned}
$$

\subsubsection{Matrix operators}

Let the $e_{i j}$ 's be the matrix unities of $\operatorname{End}\left(\mathbb{C}^{N}\right)$. Using these one can build the permutation operator

$$
\mathbb{P}=\sum_{i j} e_{i j} \otimes e_{j i}
$$

For the $\mathfrak{s o}_{N}$ models one can build the trace operator

$$
\mathbb{K}=\sum_{i j} e_{i j} \otimes\left(e_{j i}\right)^{t}=\sum_{i j} e_{i j} \otimes e_{-i,-j},
$$

where we defined the transposition $\left(e_{i, j}\right)^{t}=e_{-j,-i}$.

Using these operators we can define the $\mathfrak{g l}_{N}$ and $\mathfrak{s o}_{N} R$-matrices

$$
\begin{aligned}
& R(u)=\mathbb{I}-\frac{\mathbb{P}}{u}, \\
& R(u)=\mathbb{I}-\frac{\mathbb{P}}{u}+\frac{\mathbb{K}}{u-\kappa},
\end{aligned}
$$

where $\kappa=N / 2-1$. The $\mathfrak{s o}_{N} R$-matrix satisfies the crossing equation

$$
R^{t}(u)=R^{t_{1}}(u)=R^{t_{2}}(u)=R(\kappa-u) .
$$

\subsubsection{Yangians and highest weight representations}

Yangian $\boldsymbol{Y}(\boldsymbol{N})$ and extended Yangian $\boldsymbol{X}\left(\mathfrak{s o}_{N}\right)$. The Yangian $Y(N)[47]$ and the extended Yangian $X\left(\mathfrak{s o}_{N}\right)[48]$ are associative algebras with generators $t_{i j}^{(k)}$ where $k \in \mathbb{Z}_{+}$ satisfying some quadratic relations. These can be written in a more compact form using the formal series

$$
t_{i j}(u)=\delta_{i j}+\sum_{k=1}^{\infty} t_{i j}^{(k)} u^{-k}
$$


and define the T-matrix according to (5.3). The defining relation of $Y(N)$ and $X\left(\mathfrak{s o}_{N}\right)$ are then given by the RTT relations (5.2), where we need to use the $\mathfrak{g l}_{N}$ and $\mathfrak{s o}_{N}$ symmetric R-matrices (5.19) respectively. These algebras also form Hopf algebras but only the coproduct is needed

$$
\Delta: t_{i j}(u) \rightarrow \sum_{a} t_{i a}(u) \otimes t_{a j}(u)
$$

There is an algebra homomorphism ev $: Y(N) \rightarrow \mathrm{U}\left(\mathfrak{g l}_{N}\right)$ such that

$$
\mathrm{ev}: t_{i j}(u) \rightarrow \delta_{i j}+u^{-1} E_{i j}
$$

where $E_{i j}$ 's are the generators of $\mathfrak{g l}_{N}$. Using the evaluation homomorphism (5.24), the $\mathfrak{g l}_{N}$ rep $L\left(\lambda_{1}, \ldots, \lambda_{N}\right)$ is also an h.w. representation of $Y(N)$ i.e.

$$
\begin{array}{ll}
t_{i j}(u) \cdot v=0, & \text { for } i<j, \\
t_{i i}(u) \cdot v=\lambda_{i}(u) v, & \text { for } i=1, \ldots, N,
\end{array}
$$

where $\lambda_{i}(u)=1+\lambda_{i} u^{-1}$. Let us use the following notation

$$
t_{i j}(u) \cdot L\left(\lambda_{1}, \ldots, \lambda_{N}\right)=\mathcal{L}_{i j}^{\left(\lambda_{1}, \ldots, \lambda_{N}\right)}(u) L\left(\lambda_{1}, \ldots, \lambda_{N}\right)
$$

The matrix $\mathcal{L}$ is the Lax operator.

Connection between $\boldsymbol{Y}(4)$ and $\boldsymbol{X}\left(\mathfrak{s o}_{6}\right)$. In contrast to the Yangian $Y(N)$, there is no surjective homomorphism from $X\left(\mathfrak{s o}_{N}\right)$ onto the algebra $\mathrm{U}\left(\mathfrak{s o}_{N}\right)$, therefore we cannot generally use $\mathfrak{s o}_{N}$ modules as $X\left(\mathfrak{s o}_{N}\right)$ modules. Nevertheless if we work only with $X\left(\mathfrak{s o}_{6}\right)$ we can use $\mathfrak{g l}_{4}$ representations since there exists a Hopf algebra homomorphism between $X\left(\mathfrak{s o}_{6}\right)$ and $Y(4)$ such that

$$
T^{\mathfrak{s o} 6}(u) \rightarrow(1-P) T_{1}^{\mathfrak{g l}_{4}}(u) T_{2}^{\mathfrak{g l} l_{4}}(u-1)=T_{2}^{\mathfrak{g l}_{4}}(u-1) T_{2}^{\mathfrak{g l} \mathfrak{l}_{4}}(u)(1-P),
$$

where $(1-P)$ is a projection of $\mathbb{C}^{4} \otimes \mathbb{C}^{4}$ to the antisymmetric subspace $\mathbb{C}^{6}$. Consider the vector space $\mathbb{C}^{4}$ with the canonical basis $e_{1}, e_{2}, e_{3}, e_{4}$ and the vector space $\mathbb{C}^{6}$ with the canonical basis $v_{-3}, v_{-2}, v_{-1}, v_{1}, v_{2}, v_{3}$. We can identify the antisymmetric subspace of $\mathbb{C}^{4} \otimes \mathbb{C}^{4}$ with $\mathbb{C}^{6}$ as

$$
\begin{array}{ll}
v_{-3}=e_{1} \otimes e_{2}-e_{2} \otimes e_{1}, & v_{3}=e_{3} \otimes e_{4}-e_{4} \otimes e_{3}, \\
v_{-2}=e_{3} \otimes e_{1}-e_{1} \otimes e_{3}, & v_{2}=e_{2} \otimes e_{4}-e_{2} \otimes e_{4}, \\
v_{-1}=e_{1} \otimes e_{4}-e_{4} \otimes e_{1}, & v_{1}=e_{2} \otimes e_{3}-e_{3} \otimes e_{2} .
\end{array}
$$

Using the evaluation homomorphism (5.24), the $\mathfrak{g l}_{4}$ module $L\left(\lambda_{1}, \lambda_{2}, \lambda_{3}, \lambda_{4}\right)$ is an $X\left(\mathfrak{s o}_{6}\right)$ module. 


\subsubsection{Twisted Yangians}

Twisted Yangian $\boldsymbol{Y}^{+}(\mathbf{3})$. Let us now define the twisted Yangian $Y^{+}(3)$ [47]. In this paragraph we assume that $i, j$ run through the set $\{-1,0,1\}$. Let us then introduce the S-matrix (5.5) which now, with the $K$-matrix given by eqs. (3.9) and (4.2), takes the explicit form

$$
S(u):=T(u) T^{t}(-u),
$$

or in terms of its matrix elements $S(u)=\left(s_{i j}(u)\right)$

$$
s_{i j}(u)=\sum_{a} t_{i a}(u) t_{-j,-a}(-u)
$$

where $t_{i j}(u)$ are the generators of $Y(3)$. The twisted Yangian $Y^{+}(3)$ is generated by $s_{i j}(u)$, therefore $Y^{+}(3)$ is a subalgebra of $Y(3)$.

Using (5.32), one can derive that the matrix $S(u)$ satisfies the quaternary relation (5.6) and additionally the symmetry relation

$$
S^{t}(-u)=S(u)+\frac{1}{2 u}(S(u)-S(-u)) .
$$

From the symmetry relation (5.34) we can then obtain that the elements

$$
s_{11}^{(k)}, s_{10}^{(k)}, s_{01}^{(k)}, s_{00}^{(2 k)}, s_{1,-1}^{(2 k)}, s_{-1,1}^{(2 k)}, \quad k=1,2, \ldots,
$$

constitute a system of linearly independent generators.

The $Y^{+}(3)$ module $V$ is highest weight if there exists a nonzero vector $v \in V$ such that $V$ is generated by $v$ and

$$
\begin{array}{ll}
s_{i j}(u) \cdot v=0, & \text { for } i<j, \\
s_{i i}(u) \cdot v=\mu_{i}(u) v, & \text { for } i=0,1 .
\end{array}
$$

In [47] it was shown that every finite dimensional irrep of $Y^{+}(3)$ is a highest weight representation (Theorem 3.3).

The mapping

$$
F_{i j} \rightarrow s_{i j}^{(1)},
$$

defines an inclusion $\mathrm{U}\left(\mathfrak{s o}_{3}\right) \rightarrow Y^{+}(3)$. We can choose the following conventions for the $\mathfrak{s o}_{3} \cong \mathfrak{s l}_{2}$ generators

$$
\begin{aligned}
S_{z} & =-F_{11}=F_{-1,-1}, \\
S_{+} & =F_{01}=-F_{-1,0}, \\
S_{-} & =F_{10}=F_{0,-1} .
\end{aligned}
$$

Using the defining equation (5.32) of $Y^{+}(3)$, the $\mathfrak{g l}_{3}$ module $L\left(\lambda_{1}, \lambda_{2}, \lambda_{3}\right)$ defines also an $Y^{+}(3)$ module. Let $\left|a_{1}, a_{2}, a_{3}\right\rangle \in L(\alpha, \beta, \gamma)$ such that

$$
E_{i i} \cdot\left|a_{1}, a_{2}, a_{3}\right\rangle=a_{i}\left|a_{1}, a_{2}, a_{3}\right\rangle .
$$


Using (5.38) and (5.39)-(5.41), we can obtain that

$$
S_{z} \cdot\left|a_{1}, a_{2}, a_{3}\right\rangle=\left(a_{1}-a_{3}\right)\left|a_{1}, a_{2}, a_{3}\right\rangle
$$

We can also see that

- the $\mathfrak{g l}_{3}$ generators $E_{1,0}$ and $E_{0,-1}$ decrease the $\mathfrak{s l}_{2}$ weight by one,

- the $\mathfrak{g l}_{3}$ generators $E_{0,1}$ and $E_{-1,0}$ increase the $\mathfrak{s l}_{2}$ weight by one,

- the $\mathfrak{g l}_{3}$ generator $E_{1,-1}$ decreases the $\mathfrak{s l}_{2}$ weight by two,

- the $\mathfrak{g l}_{3}$ generators $E_{-1,1}$ increases the $\mathfrak{s l}_{2}$ weight by two.

In the following we will use the $\mathfrak{g l}_{3}$ module $L\left(\lambda_{1}, \lambda_{1}, \lambda_{2}\right)$ which is a highest weight rep of $Y^{+}(3)$ with highest weight

$$
\begin{aligned}
& \mu_{1}(u)=\left(1+\lambda_{2} u^{-1}\right)\left(1-\lambda_{1} u^{-1}\right) \\
& \mu_{0}(u)=\left(1+\lambda_{1} u^{-1}\right)\left(1-\lambda_{1} u^{-1}\right) .
\end{aligned}
$$

Using the Lax operator we can write

$$
s_{i j}(u) \cdot v=\sum_{a} \mathcal{L}_{i, a}^{\left(\lambda_{1}, \lambda_{1}, \lambda_{2}\right)}(u) \mathcal{L}_{i,-a}^{\left(\lambda_{1}, \lambda_{1}, \lambda_{2}\right)}(-u) v
$$

for all $v \in L\left(\lambda_{1}, \lambda_{1}, \lambda_{2}\right)$.

From the coproduct of $Y(3)$ (5.23), one can show that the twisted Yangian $Y^{+}(3)$ is a coideal subalgebra of $Y(3)$ i.e.

$$
\Delta\left(s_{i j}(u)\right)=\sum_{a b} t_{i a}(u) t_{-j,-b}(-u) \otimes s_{a b}(u) \quad \in Y(3) \otimes Y^{+}(3) .
$$

Using this equation, any tensor product $L \otimes V$ of a $Y(3)$ module $L$ and a $Y^{+}(3)$ module $V$ is a $Y^{+}(3)$ representation i.e.

$$
y \cdot(v \otimes w)=\Delta(y)(v \otimes w)
$$

where $y \in Y^{+}(3), v \in L$ and $w \in V$.

Extended twisted Yangian $\boldsymbol{X}\left(\mathfrak{s o}_{6}, \mathfrak{s o}_{5}\right)$. Let us again introduce the S-matrix (5.5) by setting

$$
S(u):=T(u) K(u) T^{t}(-u)
$$


where $T(u)$ is the generating function of $X\left(\mathfrak{s o}_{6}\right)$ and the K-matrix is explicitly given by

$$
K(u)=\left(\begin{array}{cccccc}
\frac{u}{u+1} & 0 & 0 & 0 & 0 & 0 \\
0 & \frac{u}{u+1} & 0 & 0 & 0 & 0 \\
0 & 0 & -\frac{1}{u+1} & 1 & 0 & 0 \\
0 & 0 & 1 & -\frac{1}{u+1} & 0 & 0 \\
0 & 0 & 0 & 0 & \frac{u}{u+1} & 0 \\
0 & 0 & 0 & 0 & 0 & \frac{u}{u+1}
\end{array}\right) .
$$

The algebra generated by $s_{i j}(u)$ is the extended twisted Yangian $X\left(\mathfrak{s o}_{6}, \mathfrak{s o}_{5}\right)$ [49], therefore $X\left(\mathfrak{s o}_{6}, \mathfrak{s o}_{5}\right)$ is a subalgebra of $X\left(\mathfrak{s o}_{6}\right)$.

The K-matrix satisfies the twisted Yang-Baxter equation (5.4) and the following symmetry equation

$$
K^{t}(u)=K(-u)-\frac{2 u}{(u+1)(u-1)} 1
$$

Using (5.21) and (5.51) one can then prove that the matrix $S(u)$ satisfies the following quaternary relation and symmetry relation

$$
\begin{aligned}
R_{12}(u-v) S_{1}(u) R_{12}(u+v+2) S_{2}(v) & =S_{2}(v) R_{12}(u+v+2) S_{1}(u) R_{12}(u-v) \\
S^{t}(u) & =S(-u)+\frac{1}{2 u}(S(u)-S(-u))-\frac{1}{2 u-2} \operatorname{tr}(S(u)) \mathbb{I}
\end{aligned}
$$

In [49] it was shown that the reflection algebras generated by (5.52) and (5.53) are isomorphic to $X\left(\mathfrak{s o}_{6}, \mathfrak{s o}_{5}\right)$ (Theorem 4.2). Unfortunately, the representation theory of $X\left(\mathfrak{s o}_{6}, \mathfrak{s o}_{5}\right)$ has hardly been studied in the literature. In $[50,51]$ only twisted Yangians with diagonal $K$-matrices were studied. Therefore the twisted Yangian $X\left(\mathfrak{s o}_{6}, \mathfrak{s o}_{5}\right)$ was ignored (see the explicit form of the $K$-matrix (5.50)). To the best of our knowledge the proper definition of the $X\left(\mathfrak{s o}_{6}, \mathfrak{s o}_{5}\right)$ highest weight representations has not yet appeared in the literature. Nevertheless, using the algebra homomorphism (5.28) and investigating the algebra embedding $\mathrm{U}\left(\mathfrak{s o}_{5}\right) \subset X\left(\mathfrak{s o}_{6}, \mathfrak{s o}_{5}\right)$ we can conjecture that the following is the correct definition for the $X\left(\mathfrak{s o}_{6}, \mathfrak{s o}_{5}\right)$ highest weight representations. The $X\left(\mathfrak{s o}_{6}, \mathfrak{s o}_{5}\right)$ module $V$ is highest weight if there exists a nonzero vector $v \in V$ such that $V$ is generated by $v$ and

$$
\begin{aligned}
s_{i j}(u) \cdot v & =0, \quad \text { for all } i<j \text { where }(i, j) \neq(-1,1), \\
s_{i i}(u) \cdot v & =\mu_{i}(u) v, \\
s_{1,-1}(u) \cdot v & =\mu^{(+)}(u) v, \\
s_{-1,1}(u) \cdot v & =\mu^{(-)}(u) v .
\end{aligned}
$$

From the symmetry relation (5.53) we can obtain that the $\mu_{i}(u)$ 's are not independent, every $\mu_{i}(u)$ can be expressed in terms of $\mu_{1}(u), \mu_{2}(u)$ and $\mu_{3}(u)$. Using the defining equation (5.49) of $X\left(\mathfrak{s o}_{6}, \mathfrak{s o}_{5}\right)$, the $\mathfrak{g l}_{4}$ module $L\left(\lambda_{1}, \lambda_{2}, \lambda_{3}, \lambda_{4}\right)$ also defines an $X\left(\mathfrak{s o}_{6}, \mathfrak{s o}_{5}\right)$ module. 


\section{$5.3 \quad(\mathrm{SU}(3), \mathrm{SO}(3))$ case}

Now let us continue with the representation which come from the MPS. We consider the (SU(3), $\mathrm{SO}(3)$ ) K-matrix as given by (4.14). From that representation we can derive the following components in the complex basis, see also [32]

$$
\begin{aligned}
\psi_{1,1}^{(s)}(u) & =1-u^{-1} S_{z}-\frac{1}{2} u^{-2}\left(s(s+1)-S_{z}\left(S_{z}+1\right)\right), \\
\psi_{0,0}^{(s)}(u) & =1-u^{-2} S_{z}^{2}, \\
\psi_{-1,-1}^{(s)}(u) & =1+u^{-1} S_{z}-\frac{1}{2} u^{-2}\left(s(s+1)+S_{z}\left(S_{z}+1\right)\right), \\
\psi_{0,-1}^{(s)}(u) & =-2 i u^{-1} S_{-}-i u^{-2} S_{z} S_{-}, \\
\psi_{1,0}^{(s)}(u) & =2 i u^{-1} S_{-}-i u^{-2} S_{-} S_{z}, \\
\psi_{1,-1}^{(s)}(u) & =u^{-2} S_{-}^{2}, \\
\psi_{-1,0}^{(s)}(u) & =2 i u^{-1} S_{+}+i u^{-2} S_{+} S_{z}, \\
\psi_{1,0}^{(s)}(u) & =-2 i u^{-1} S_{+}+i u^{-2} S_{z} S_{+}, \\
\psi_{1,-1}^{(s)}(u) & =u^{-2} S_{+}^{2},
\end{aligned}
$$

where $S_{x}^{2}+S_{y}^{2}+S_{z}^{2}=s(s+1)$. This is a $k=2 s+1$ dimensional irreducible representation of the twisted Yangian $Y^{+}(3)$. Let us denote it $V(s)$. The $Y^{+}(3)$ highest weights of $V(s)$ are

$$
\begin{aligned}
& \mu_{1}(u)=\left(1-s u^{-1}\right), \\
& \mu_{0}(u)=\left(1-s^{2} u^{-2}\right) .
\end{aligned}
$$

From (5.44) and (5.45) we can see that $V(s)$ can be embedded into $L\left(\lambda_{1}, \lambda_{1}, \lambda_{2}\right)$ if $\lambda_{1}=s$ and $\lambda_{2}=0$ but $L(s, s, 0)$ is finite dimensional iff $s \in \mathbb{N}$, therefore we only have a chance to find a connection between $\left|\Psi_{\delta}\right\rangle$ and $\left|\mathrm{MPS}_{k}\right\rangle$ when $k$ is odd.

For even $k$ we have to use tensor product representations (see (5.47) and (5.48)). The representation $L\left(\lambda_{1}, \lambda_{1}, \lambda_{2}\right) \otimes V(1 / 2)$ has highest weight

$$
\begin{aligned}
& \mu_{1}(u)=\left(1+\lambda_{2} u^{-1}\right)\left(1-\lambda_{1} u^{-1}\right)\left(1-\frac{1}{2} u^{-1}\right), \\
& \mu_{0}(u)=\left(1-\lambda_{1}^{2} u^{-2}\right)\left(1-\frac{1}{4} u^{-1}\right),
\end{aligned}
$$

we can see that $V(s)$ can be embedded into $L\left(\lambda_{1}, \lambda_{1}, \lambda_{2}\right) \otimes V(1 / 2)$ if $\lambda_{1}=s$ and $\lambda_{2}=1 / 2$.

\subsubsection{Odd $k=2 s+1$}

We have seen that the even and odd $k$ cases must be treated differently. Let us start with the odd case. We can show that $V(s)$ is embedded into $L(s, s, 0)$ where $s \in \mathbb{Z}_{+}$for small $s$. These calculations can be found in appendix B. Using these explicit results we conjecture the embedding for general $s$ the twisted Yangian $Y^{+}(3)$ acts on $L(s, s, 0) \cong V(s) \oplus L(s, s, 2)$ as

$$
\begin{aligned}
s_{i j}(u) \cdot v_{1} & =\mathcal{L}_{i a}^{(s, s, 0)}(u) \mathcal{L}_{-j,-a}^{(s, s, 0)}(-u) v_{1}= \\
& =\left(\begin{array}{cc}
\psi_{i, j}^{(s)}(u) & X \\
0 & \mathcal{L}_{i a}^{(s, s, 2)}(u) \mathcal{L}_{-j,-b}^{(s, s, 2)}(-u)
\end{array}\right)\left(\begin{array}{l}
w_{1} \\
w_{2}
\end{array}\right),
\end{aligned}
$$


for all $y \in Y^{+}(3)$ where $v \in L(s, s, 0), w_{1} \in V(s), w_{2} \in L(s, s, 2)$ and $s \in \mathbb{Z}_{>1}$. In (5.71) we used the following conjecture.

Conjecture 1. $L(s, s, 2)$ is an irrep of $Y^{+}(3)$ for all $s>1$.

Ratio of the overlaps. In the following we will show that these results are consistent with the overlap formulas (4.12) and (4.13). So far, we used the convention (5.19) for the $R$-matrix but now we switch to the slightly different convention

$$
\tilde{R}(u)=u \mathbb{I}+i \mathbb{P},
$$

and hence use rescaled matrices:

$$
\begin{aligned}
\tilde{s}_{i, j}(u) & =u^{2} s_{i, j}(i u), \\
\tilde{t}_{i, j}(u) & =u t_{i, j}(i u) .
\end{aligned}
$$

Let $\rho$ be a representation of the twisted Yangian and let us define the spectral parameter independent matrix

$$
\phi_{i j}=\rho\left(s_{i k}(0)\right) C_{k j}=\rho\left(s_{i,-j}(0)\right),
$$

where $C$ is the charge conjugation matrix. Furthermore, let us define the following state

$$
|\Psi\rangle=\sum_{i_{1, j_{1}, \ldots i_{L / 2}, j_{L / 2}}} \operatorname{tr}_{A}\left[\phi_{i_{1} j_{1}} \ldots \phi_{i_{L / 2} j_{L / 2}}\right]\left|i_{1}, j_{1}, \ldots, i_{L / 2}, j_{L / 2}\right\rangle
$$

For the trivial representation

$$
\rho\left(\tilde{s}_{i j}(u)\right)=\delta_{i, j},
$$

and $|\Psi\rangle=\left|\Psi_{\delta}\right\rangle$ given in eq. (4.1). For representations $L(s, s, m)$ and $V(s)$ we can obtain the following states

$$
\begin{aligned}
& \sum \operatorname{tr}_{A}\left[\tilde{\mathcal{L}}_{i_{1} a_{1}}^{(s, s, m)}(0) \tilde{\mathcal{L}}_{j_{1},-a_{1}}^{(s, s, m)}(0) \ldots \tilde{\mathcal{L}}_{i_{L / 2} a_{L / 2}}^{(s, s, m)}(0) \tilde{\mathcal{L}}_{j_{L / 2},-a_{L / 2}}^{(s, s, m)}(0)\right]\left|i_{1}, j_{1}, \ldots, i_{L / 2}, j_{L / 2}\right\rangle, \\
& \sum \operatorname{tr}_{A}\left[S_{i_{1}} S_{j_{1}} \ldots S_{i_{L / 2}} S_{j_{L / 2}}\right]\left|i_{1}, j_{1}, \ldots, i_{L / 2}, j_{L / 2}\right\rangle .
\end{aligned}
$$

Therefore the equation (5.71) connects $\left|\mathrm{MPS}_{2 s+1}\right\rangle$ to the delta-state $\left|\Psi_{\delta}\right\rangle$ as

$$
\left|\mathrm{MPS}_{2 s+1}\right\rangle=\left(\tilde{T}^{(s, s, 0)}(0)-\tilde{T}^{(s, s, 2)}(0)\right)\left|\Psi_{\delta}\right\rangle,
$$

where

$$
\begin{aligned}
& \tilde{T}^{(s, s, m)}(u)=\operatorname{Tr}_{0}\left[\mathcal{L}_{01}^{(s, s, m)}(u) \ldots \mathcal{L}_{0 L}^{(s, s, m)}(u)\right], \\
& \tilde{\mathcal{L}}^{(s, s, m)}(u)=\tilde{\mathcal{L}}_{i, j}^{(s, s, m)}(u) \otimes e_{i, j}=u \mathbb{I}-i E_{i, j}^{(s, s, m)} \otimes e_{i, j}=u \mathbb{I}+i E_{i, j}^{(-m,-s,-s)} \otimes e_{j, i},
\end{aligned}
$$

where we have used the fact that $E_{i, j} \rightarrow-E_{j, i}$ is a Lie algebra automorphism connecting a representation to its contra-gradient. Let us use another notation:

$$
\begin{aligned}
& \tilde{\mathcal{L}}^{(s)}(u)=u-i \frac{s-1}{2}+i E_{i, j}^{(s, 0,0)} \otimes e_{j, i}, \\
& \tilde{T}^{(s)}(u)=\operatorname{Tr}_{0}\left[\tilde{\mathcal{L}}_{01}^{(s)}(u) \ldots \tilde{\mathcal{L}}_{0 L}^{(s)}(u)\right] .
\end{aligned}
$$


From (5.82) and (5.83) we can see that

$$
\begin{aligned}
& \tilde{\mathcal{L}}^{(s, s, 0)}(u)=\tilde{\mathcal{L}}^{(s)}\left(u-i \frac{s+1}{2}\right), \\
& \tilde{\mathcal{L}}^{(s, s, 2)}(u)=\tilde{\mathcal{L}}^{(s-2)}\left(u-i \frac{s+3}{2}\right),
\end{aligned}
$$

therefore

$$
\left|\mathrm{MPS}_{2 s+1}\right\rangle=\left(\tilde{T}^{(s)}\left(-i \frac{s+1}{2}\right)-\tilde{T}^{(s-2)}\left(-i \frac{s+3}{2}\right)\right)\left|\Psi_{\delta}\right\rangle,
$$

and the ratio of the overlaps is equal to the difference of eigenvalues of the transfer matrices

$$
\frac{\left\langle\mathrm{MPS}_{2 s+1} \mid \mathbf{u}\right\rangle}{\left\langle\Psi_{\delta} \mid \mathbf{u}\right\rangle}=\tilde{T}^{(s)}\left(-i \frac{s+1}{2}\right)-\tilde{T}^{(s-2)}\left(-i \frac{s+3}{2}\right) .
$$

The eigenvalues of the transfer matrices can be written as [52]

$$
\begin{aligned}
\tilde{T}^{(s)}(u)= & Q_{0}\left(-i u-\frac{s}{2}\right) Q_{+}\left(-i u+\frac{s+3}{2}\right) \sum_{k=0}^{s} \frac{\left(u+i \frac{s+1}{2}-i k\right)^{L} Q_{+}\left(-i u+\frac{s+1}{2}-k\right)}{Q_{0}\left(-i u+\frac{s}{2}-k\right) Q_{0}\left(-i u+\frac{s+2}{2}-k\right)} \\
& \times \sum_{l=0}^{k} \frac{Q_{0}\left(-i u+\frac{s+2}{2}-l\right)}{Q_{+}\left(-i u+\frac{s+1}{2}-l\right) Q_{+}\left(-i u+\frac{s+3}{2}-l\right)} .
\end{aligned}
$$

Let us assume that $L, N_{0}, N_{+}$are even, then

$$
\begin{aligned}
\tilde{T}^{(s)}\left(-i \frac{s+1}{2}\right)-\tilde{T}^{(s-2)}\left(-i \frac{s+3}{2}\right)= \\
=Q_{0}\left(s+\frac{1}{2}\right) Q_{+}(1) \sum_{k=0}^{s}(i k)^{L} \frac{Q_{+}(k)}{Q_{0}\left(k+\frac{1}{2}\right) Q_{0}\left(k-\frac{1}{2}\right)} \sum_{l=0}^{k} \frac{Q_{0}\left(l-\frac{1}{2}\right)}{Q_{+}(l) Q_{+}(l-1)}- \\
\quad-Q_{0}\left(s+\frac{1}{2}\right) Q_{+}(1) \sum_{k=0}^{s-2}(i k+i 2)^{L} \frac{Q_{+}(k+2)}{Q_{0}\left(k+\frac{5}{2}\right) Q_{0}\left(k+\frac{3}{2}\right)} \sum_{l=0}^{k} \frac{Q_{0}\left(l+\frac{3}{2}\right)}{Q_{+}(l+2) Q_{+}(l+1)}= \\
=\frac{Q_{0}\left(\frac{1}{2}\right)}{Q_{+}(0)} Q_{0}\left(s+\frac{1}{2}\right) \sum_{k=1}^{s} 2(i k)^{L} \frac{Q_{+}(k)}{Q_{0}\left(k+\frac{1}{2}\right) Q_{0}\left(k-\frac{1}{2}\right)},
\end{aligned}
$$

i.e.

$$
\tilde{T}^{(s)}\left(-i \frac{s+1}{2}\right)-\tilde{T}^{(s-2)}\left(-i \frac{s+3}{2}\right)=\frac{Q_{0}\left(\frac{1}{2}\right)}{Q_{+}(0)} \mathbb{T}_{2 s}(0),
$$

which is consistent with

$$
\left\langle\Psi_{\delta} \mid \mathbf{u}\right\rangle=\frac{Q_{+}(0)}{Q_{0}\left(\frac{1}{2}\right)} \sqrt{\frac{Q_{0}(0) Q_{0}\left(\frac{1}{2}\right)}{\bar{Q}_{+}(0) \bar{Q}_{+}\left(\frac{1}{2}\right)}} \sqrt{\frac{\operatorname{det} G_{+}}{\operatorname{det} G_{-}}},
$$

and

$$
\left\langle\mathrm{MPS}_{2 s+1} \mid \mathbf{u}\right\rangle=\mathbb{T}_{2 s}(0) \sqrt{\frac{Q_{0}(0) Q_{0}\left(\frac{1}{2}\right)}{\bar{Q}_{+}(0) \bar{Q}_{+}\left(\frac{1}{2}\right)}} \sqrt{\frac{\operatorname{det} G_{+}}{\operatorname{det} G_{-}}} .
$$




\subsubsection{Even $k=2 s+1$}

Let us continue with the representations $L(s, s, 1 / 2) \otimes V(1 / 2)$ where $s=\frac{3}{2}, \frac{5}{2}, \ldots$. For general $s$, the twisted Yangian $Y^{+}(3)$ acts on $L(s, s, 1 / 2) \otimes V(1 / 2) \cong V(s) \oplus(L(s, s, 2) \otimes V(1 / 2))$ as

$$
\begin{aligned}
& s_{i j}(u) \cdot\left(v_{1} \otimes v_{2}\right)=\mathcal{L}_{i a}^{(s, s, 1 / 2)}(u) \mathcal{L}_{-j,-b}^{(s, s, 1 / 2)}(-u) v_{1} \otimes \psi_{a, b}^{(1 / 2)}(u) v_{2}= \\
& \quad=\left(\begin{array}{cc}
\left(1-\frac{1}{4} u^{-2}\right) \psi_{i, j}^{(s)}(u) & X \\
0 & \mathcal{L}_{i a}^{(s, s, 3 / 2)}(u) \mathcal{L}_{-j,-b}^{(s, s, 3 / 2)}(-u) \otimes \psi_{a, b}^{(1 / 2)}(u)
\end{array}\right)\left(\begin{array}{c}
w_{1} \\
w_{2} \otimes w_{3}
\end{array}\right),
\end{aligned}
$$

for all $v_{1} \in L(s, s, 1 / 2), v_{2}, w_{3} \in V(1 / 2), w_{1} \in V(s), w_{2} \in L(s, s, 3 / 2)$ and $s \in \mathbb{Z}_{+}+1 / 2$. We used the following conjecture.

Conjecture 2. $L(s, s, 3 / 2) \otimes V(1 / 2)$ is an irrep of $Y^{+}(3)$ for all $s \in \mathbb{Z}_{+}+\frac{1}{2}$.

See appendix B for the explanation.

Ratio of the overlaps. In the following let us check that these results are consistent with the overlap formulas. The equation (5.94) connects $\left|\mathrm{MPS}_{2 s+1}\right\rangle$ to $\left|\mathrm{MPS}_{2}\right\rangle$ as

$$
\left|\mathrm{MPS}_{2 s+1}\right\rangle=\left(\frac{2}{i}\right)^{L}\left(\tilde{T}^{(s, s, 1 / 2)}(0)-\tilde{T}^{(s, s, 3 / 2)}(0)\right)\left|\mathrm{MPS}_{2}\right\rangle
$$

where the factor $(2 / i)^{L}$ comes from the prefactor of $\psi_{i, j}^{(s)}(u)$ in (5.94). From (5.82) and (5.83) we can see that

$$
\begin{aligned}
& \tilde{\mathcal{L}}^{(s, s, 1 / 2)}(u)=\tilde{\mathcal{L}}^{(m)}\left(u-i \frac{m+2}{2}\right), \\
& \tilde{\mathcal{L}}^{(s, s, 3 / 2)}(u)=\tilde{\mathcal{L}}^{(m-1)}\left(u-i \frac{m+3}{2}\right),
\end{aligned}
$$

where

$$
m=s-1 / 2 \text {. }
$$

Therefore

$$
\left|\operatorname{MPS}_{2 s+1}\right\rangle=\left(\frac{2}{i}\right)^{L}\left(\tilde{T}^{(m)}\left(-i \frac{m+2}{2}\right)-\tilde{T}^{(m-1)}\left(-i \frac{m+3}{2}\right)\right)\left|\operatorname{MPS}_{2}\right\rangle,
$$

and from the ratio of the overlaps we have to obtain that

$$
\frac{\left\langle\mathrm{MPS}_{2 s+1} \mid \mathbf{u}\right\rangle}{\left\langle\mathrm{MPS}_{2} \mid \mathbf{u}\right\rangle}=\left(\frac{2}{i}\right)^{L}\left(\tilde{T}^{(m)}\left(-i \frac{m+2}{2}\right)-\tilde{T}^{(m-1)}\left(-i \frac{m+3}{2}\right)\right) \stackrel{?}{=} \frac{\mathbb{T}_{2 s}(0)}{\mathbb{T}_{1}(0)}
$$


Substituting (5.89):

$$
\begin{aligned}
\tilde{T}^{(m)}\left(-i \frac{m+2}{2}\right)-\tilde{T}^{(m-1)}\left(-i \frac{m+3}{2}\right)= \\
=Q_{0}(m+1) Q_{+}\left(\frac{1}{2}\right) \sum_{k=0}^{m}\left(i k+\frac{i}{2}\right)^{L} \frac{Q_{+}\left(k+\frac{1}{2}\right)}{Q_{0}(k+1) Q_{0}(k)} \sum_{l=0}^{k} \frac{Q_{0}(l)}{Q_{+}\left(l+\frac{1}{2}\right) Q_{+}\left(l-\frac{1}{2}\right)}- \\
\quad-Q_{0}(m+1) Q_{+}\left(\frac{1}{2}\right) \sum_{k=0}^{m-1}\left(i k+\frac{3 i}{2}\right)^{L} \frac{Q_{+}\left(k+\frac{3}{2}\right)}{Q_{0}(k+2) Q_{0}(k+1)} \sum_{l=0}^{k} \frac{Q_{0}(l+1)}{Q_{+}\left(l+\frac{3}{2}\right) Q_{+}\left(l+\frac{1}{2}\right)}= \\
=\frac{Q_{0}(0)}{Q_{+}\left(\frac{1}{2}\right)} Q_{0}\left(s+\frac{1}{2}\right) \sum_{k=1 / 2}^{s}(i k)^{L} \frac{Q_{+}(k)}{Q_{0}\left(k+\frac{1}{2}\right) Q_{0}\left(k-\frac{1}{2}\right)} .
\end{aligned}
$$

Using the explicit forms of $\mathbb{T}_{2 s}(0)$ and $\mathbb{T}_{1}(0)$

$$
\begin{aligned}
\mathbb{T}_{2 s}(0) & =Q_{0}\left(s+\frac{1}{2}\right) \sum_{k=1 / 2}^{s} 2(i k)^{L} \frac{Q_{+}(k)}{Q_{0}\left(k+\frac{1}{2}\right) Q_{0}\left(k-\frac{1}{2}\right)}, \\
\mathbb{T}_{1}(0) & =2\left(\frac{i}{2}\right)^{L} \frac{Q_{+}\left(\frac{1}{2}\right)}{Q_{0}(0)}
\end{aligned}
$$

we just obtained that

$$
\left(\frac{2}{i}\right)^{L}\left(\tilde{T}^{(m)}\left(-i \frac{m+2}{2}\right)-\tilde{T}^{(m-1)}\left(-i \frac{m+3}{2}\right)\right)=\frac{\mathbb{T}_{2 s}(0)}{\mathbb{T}_{1}(0)}
$$

\section{$5.4(\mathrm{SO}(6), \mathrm{SO}(5))$ case}

The MPS can be built from the K-matrix $\tilde{K}=e_{a b} \otimes \psi_{a b}+e_{66} \otimes \psi_{66}$, where $\psi_{a b}$ and $\psi_{66}$ are given in equation (3.12) and (3.13). In the twisted Yangian language we use different normalization and basis. After the normalization

$$
S(u)=-\frac{1}{4} u^{-3}\left(1-u^{-1}\right) \tilde{K}(u),
$$

and basis changing the S-matrix satisfies the reflection equation (5.52) and the symmetry relation (5.53). This is a highest weight representation and let us denote it by $V(n)$. From the explicit forms

$$
\begin{aligned}
s_{3,3}(u) & =\tilde{g}_{1}(u) \tilde{G}_{1} \tilde{G}_{-1}+\tilde{g}_{2}(u)\left[\tilde{G}_{1}, \tilde{G}_{-1}\right]+\tilde{f}(u), \\
s_{2,2}(u) & =\tilde{g}_{1}(u) \tilde{G}_{2} \tilde{G}_{-2}+\tilde{g}_{2}(u)\left[\tilde{G}_{2}, \tilde{G}_{-2}\right]+\tilde{f}(u), \\
s_{1,1}(u) & =\frac{1}{2}\left(\tilde{g}_{1}(u) \tilde{G}_{0}^{2}+\tilde{f}(u)+\tilde{h}(u)\right), \\
s_{1,-1}(u) & =\frac{1}{2}\left(\tilde{g}_{1}(u) \tilde{G}_{0}^{2}+\tilde{f}(u)-\tilde{h}(u)\right)=s_{-1,1}(u),
\end{aligned}
$$

we calculate the weights $\mu_{3}(u), \mu_{2}(u), \mu_{1}(u)$ and $\mu^{(+)}(u)=\mu^{(-)}(u)$. We used the notation

$$
\begin{aligned}
\tilde{G}_{ \pm 1} & =\frac{1}{\sqrt{2}}\left(G_{1} \pm i G_{2}\right), \\
\tilde{G}_{ \pm 2} & =\frac{1}{\sqrt{2}}\left(G_{3} \pm i G_{4}\right), \\
\tilde{G}_{0} & =G_{5}
\end{aligned}
$$


and

$$
\begin{aligned}
\tilde{g}_{1}(u) & =-\frac{1}{2} u^{-2}\left(1-u^{-2}\right), \\
\tilde{g}_{2}(u) & =\frac{1}{2} u^{-1}\left(1-u^{-2}\right), \\
\tilde{f}(u) & =\left(1-u^{-1}\right)\left(1+\frac{C}{4} u^{-2}\right), \\
\tilde{h}(u) & =-\left(1-u^{-1}\right)\left(1+2 u^{-1}-\frac{C}{4} u^{-2}\right) .
\end{aligned}
$$

We can also calculate the highest weights of

$$
S^{D}(u)=T^{\mathfrak{s 0}_{6}}(u) K(u)\left(T^{\mathfrak{s o}_{6}}\right)^{t}(-u),
$$

for the $\mathfrak{g l}_{4}$ module $L\left(\lambda_{1}, \lambda_{2}, \lambda_{3}, \lambda_{4}\right)$. From the explicit calculation (see appendix C) we obtain that $V(n) \cong L(1+n / 2,1+n / 2,1+n / 2,1-n / 2)$, i.e.

$$
S(u)=\frac{\left(1-u^{-2}\right)^{2}}{1-\left(\frac{n}{2}+1\right)^{2} u^{-2}} T^{\mathfrak{s o}_{6}}(u) K(u)\left(T^{\mathfrak{s o}_{6}}\right)^{t}(-u),
$$

which implies that (with proper normalization of the states)

$$
\left|\operatorname{MPS}_{n}\right\rangle=\lim _{u \rightarrow 0}(2 i)^{L} t_{n}^{(1)}(u)\left|\Psi_{0}\right\rangle,
$$

where $t_{n}^{(a)}(u)$ is a solution of the Hirota equation (3.21). Let us use the z-functions

$$
\begin{aligned}
& z_{1}(u)=\frac{\left(u+\frac{i}{2}\right)^{L}}{\left(u-\frac{i}{2}\right)^{L}} \frac{Q_{-}\left(-i u-\frac{3}{2}\right)}{Q_{-}\left(-i u-\frac{1}{2}\right)}, \\
& z_{2}(u)=\frac{\left(u+\frac{i}{2}\right)^{L}}{\left(u-\frac{i}{2}\right)^{L}} \frac{Q_{0}(-i u-1)}{Q_{0}(-i u)} \frac{Q_{-}\left(-i u+\frac{1}{2}\right)}{Q_{-}\left(-i u-\frac{1}{2}\right)}, \\
& z_{3}(u)=\frac{Q_{0}(-i u+1)}{Q_{0}(-i u)} \frac{Q_{+}\left(-i u-\frac{1}{2}\right)}{Q_{+}\left(-i u+\frac{1}{2}\right)}, \\
& z_{4}(u)=\frac{Q_{+}\left(-i u+\frac{3}{2}\right)}{Q_{+}\left(-i u+\frac{1}{2}\right)} .
\end{aligned}
$$

Using the tableau sum (3.27) the eigenvalues $t_{n}^{(1)}(u)$ are then found to be

$$
\begin{aligned}
t_{n}^{(1)}(u)= & \frac{Q_{-}\left(-i u-\frac{n}{2}-1\right) Q_{+}\left(-i u+\frac{n}{2}+1\right)}{\left(u-i \frac{n}{2}\right)^{L}} \times \\
& \times \sum_{q=-n / 2}^{n / 2}(u+i q)^{L} \frac{Q_{-}(-i u+q) Q_{+}(-i u+q)}{Q_{0}\left(-i u+q-\frac{1}{2}\right) Q_{0}\left(-i u+q+\frac{1}{2}\right)} \times \\
& \times\left[\sum_{p=-n / 2}^{q} \frac{Q_{0}\left(-i u+p-\frac{1}{2}\right)}{Q_{-}(-i u+p-1) Q_{-}(u+p)}\right] \times\left[\sum_{r=q}^{n / 2} \frac{Q_{0}\left(-i u+r+\frac{1}{2}\right)}{Q_{+}(-i u+r) Q_{+}(-i u+r+1)}\right] .
\end{aligned}
$$




\section{Conclusion and outlook}

With the present work we have reached a complete understanding of the integrability properties of a class of spin chain boundary states which among other things can be used for the calculation of one-point functions in domain wall versions of $\mathcal{N}=4$ SYM theory. The boundary states in question take the form of matrix product states generated by matrices which are related to the generators of some irreducible representation of a Lie group. Matrix product states were in [5] characterized as integrable if annihilated by the odd charges of the underlying integrable spin chain. Furthermore, it was argued that this criterion being fulfilled would imply the existence of a boundary reflection matrix which together with the bulk $R$-matrix would fulfill a boundary Yang-Baxter relation which again should in principle make it possible to compute a number of quantum observables of the system in a closed form. The integrability criterion could immediately be shown to be fulfilled for two out of the three known relevant defect versions of $\mathcal{N}=4 \mathrm{SYM}$, namely the one dual to the D3-D5 probe brane system with flux and the one dual to the $\mathrm{SO}(5)$ symmetric D3-D7 probe brane system with non-vanishing instanton number [13], cf. table 1, and a closed formula for the one-point functions of the former case could be found [13].

A gap in the understanding was the apparent lack of a closed formula for one-point functions of the integrable D3-D7 probe brane setup as well as the lack of an analytical method for the derivation of these one-point functions in all but the simplest cases for the D3-D5 setup. With the present paper we have filled these gaps. First of all we have obtained an understanding of the integrability properties for the D3-D7 probe brane setup in a scattering picture by explicitly finding the appropriate boundary reflection matrix for any irreducible representation of $\mathfrak{s o}(5)$ of the relevant type, cf. eqs. (3.9) and (3.12)-(3.13). The corresponding boundary reflection matrix for the D3-D5 probe brane setup was found in [8], see also [32]. Secondly we have explicitly derived the overlap formula (2.16) and (2.18) for the D3-D7 probe brane case by starting from a simple integrable one-site state and making use of the representation theory of twisted Yangians. We have also shown that a similar approach makes it possible to prove the formula for the D3-D5 case, earlier presented without proof [13], although for simplicity we completed the proof only for the SU(3) sector. Interestingly, the derivation seems to be more involved for the supersymmetric D3-D5 case with $\mathrm{SO}(3)$ symmetric vevs than the non-supersymmetric D3-D7 with $\mathrm{SO}(5)$ symmetric vevs.

From the point of view of theoretical high energy physics, it would be extremely interesting if, using symmetries, one could bootstrap the integrable boundary reflection matrices to higher loop orders of $\mathcal{N}=4 \mathrm{SYM}$, as has been done for the bulk $S$-matrix [53], and derive the appropriate overlap formulas to all loop orders. In reference $[54,55]$ a bootstrapping procedure was shown to work for a simpler reflection matrix (without additional internal matrix structure) occurring in the study of three-point functions of two determinant operators and one single-trace operator. For our case one would expect that again a combination of the thermodynamical Bethe ansatz approach and the representation theory of twisted Yangians should be the correct way forward. A challenge is of course in the first place to take the present calculations beyond the scalar section of $\mathcal{N}=4 \mathrm{SYM}$ which is not closed at higher loop orders. An argument in favour of integrability of the defect systems 
at higher loop orders is that an exact expression for one-loop one-point functions in the $\mathrm{SU}(2)$ subsector of the D3-D5 probe-brane setup has been found and a possible asymptotic form of these has been presented [26].

Our overlap formula pertains to highest weight Bethe eigenstates but should also contain information about overlaps involving descendent states, accessible via an appropriate limiting procedure. One-point functions of descendent operators and their relevance for the boundary conformal bootstrap program was discussed for the $\mathrm{SU}(2)$ sub-sector of the D3-D5 probe brane set-up in [56].

\section{Acknowledgments}

MdL was supported by SFI, the Royal Society and the EPSRC for funding under grants UF160578, RGF $\backslash \mathrm{EA} \backslash 181011, \mathrm{RGF} \backslash \mathrm{EA} \backslash 180167$ and 18/EPSRC/3590. C.K. was supported in part by DFF-FNU through the grant DFF-FNU 4002-00037. Furthermore, we thank Jan Ambjørn for giving us access to his computer system. The research of G.L. has received funding from the Hellenic Foundation for Research and Innovation (HFRI) and the General Secretariat for Research and Technology (GSRT), in the framework of the first post-doctoral researchers support, under grant agreement No. 2595. G.L. is thankful to the School of Mathematics of Trinity College Dublin for its hospitality and generous support. T.G. was supported in part by NKFIH grant K116505. B.P. was supported by the BMENanotechnology FIKP grant (BME FIKP-NAT), by the National Research Development and Innovation Office (NKFIH) (K-2016 grant no. 119204), by the János Bolyai Research Scholarship of the Hungarian Academy of Sciences, and by the ÚNKP-19-4 New National Excellence Program of the Ministry for Innovation and Technology.

\section{A Limiting formulas for the overlaps}

As we have already mentioned the determinant formula (2.18) is strictly speaking valid only for even values of $(n+1) N_{0} / 2$. When $(n+1) N_{0} / 2$ is odd, one auxiliary Bethe root at each level vanishes and a $0 / 0$ ambiguity arises. The indeterminate form is then treated in a standard way by noting that the terms in the square brackets of (2.18) become singular for $q=0, \pm 1$. In this case we obtain:

$$
\begin{aligned}
\Lambda_{n}= & 2^{L} \sum_{q=-\frac{n}{2}}^{\frac{n}{2}}\left[q^{L} \tilde{\Lambda}_{n}^{-}(q) \tilde{\Lambda}_{n}^{+}(q)+|q|^{L}\left[1+\frac{q}{|q|}\right] \frac{Q_{0}\left(\frac{1}{2}\right) Q_{-}(q) Q_{-}\left(\frac{n}{2}+1\right)}{Q_{0}\left(q-\frac{1}{2}\right) Q_{-}(1) \bar{Q}_{-}(0)}\left[\frac{d}{d u} \ln \frac{Q_{0}\left(\frac{1}{2}\right)}{Q_{-}(1)}\right]\right. \\
& \left.\cdot \tilde{\Lambda}_{n}^{+}(q)+(-|q|)^{L}\left[1-\frac{q}{|q|}\right] \frac{Q_{0}\left(\frac{1}{2}\right) Q_{+}(q) Q_{+}\left(\frac{n}{2}+1\right)}{Q_{0}\left(q+\frac{1}{2}\right) Q_{+}(1) \bar{Q}_{+}(0)}\left[\frac{d}{d u} \ln \frac{Q_{0}\left(\frac{1}{2}\right)}{Q_{+}(1)}\right] \tilde{\Lambda}_{n}^{-}(q)\right],
\end{aligned}
$$

where

$$
\begin{aligned}
& \tilde{\Lambda}_{n}^{+}(q) \equiv \sum_{\substack{r=q \\
r \neq 0,-1}}^{n / 2} \frac{Q_{0}\left(r+\frac{1}{2}\right) Q_{+}(q) Q_{+}\left(\frac{n}{2}+1\right)}{Q_{0}\left(q+\frac{1}{2}\right) Q_{+}(r+1) Q_{+}(r)}, \\
& \tilde{\Lambda}_{n}^{-}(q) \equiv \sum_{\substack{r=-n / 2 \\
r \neq 0,1}}^{q} \frac{Q_{0}\left(r-\frac{1}{2}\right) Q_{-}(q) Q_{-}\left(\frac{n}{2}+1\right)}{Q_{0}\left(q-\frac{1}{2}\right) Q_{-}(r) Q_{-}(r-1)}
\end{aligned}
$$




\begin{tabular}{|rll|}
\hline$S_{z}=2$ & $|2,2,0\rangle$ & \\
1 & $|2,1,1\rangle$ & \\
0 & $|2,0,2\rangle$ & $|1,2,1\rangle$ \\
-1 & $|1,1,2\rangle$ & \\
-2 & $|0,2,2\rangle$ & \\
\hline
\end{tabular}

Table 2. The states of $L(2,2,0)$.

Eq. (A.1) can also be cast in the following form:

$$
\begin{aligned}
\Lambda_{n}=2^{L} \cdot\{ & \sum_{q=0}^{\frac{n}{2}} q^{L} \tilde{\Lambda}_{n}^{+}(q)\left[\tilde{\Lambda}_{n}^{-}(q)+\frac{Q_{0}\left(\frac{1}{2}\right) Q_{-}(q) Q_{-}\left(\frac{n}{2}+1\right)}{Q_{0}\left(q-\frac{1}{2}\right) Q_{-}(1) \bar{Q}_{-}(0)} \cdot \frac{d}{d u} \ln \left[\frac{Q_{0}\left(\frac{1}{2}\right)}{Q_{-}(1)}\right]^{2}\right]+ \\
& \left.+\sum_{q=-\frac{n}{2}}^{0} q^{L} \tilde{\Lambda}_{n}^{-}(q)\left[\tilde{\Lambda}_{n}^{+}(q)+\frac{Q_{0}\left(\frac{1}{2}\right) Q_{+}(q) Q_{+}\left(\frac{n}{2}+1\right)}{Q_{0}\left(q+\frac{1}{2}\right) Q_{+}(1) \bar{Q}_{+}(0)} \cdot \frac{d}{d u} \ln \left[\frac{Q_{0}\left(\frac{1}{2}\right)}{Q_{+}(1)}\right]^{2}\right]\right\}
\end{aligned}
$$

The determinant formula (2.18) has been thoroughly checked to 50 digits of accuracy for many states of various lengths $L, N_{0}=2, \ldots, 10$ and $n=1, \ldots, 8$, such that $(n+1) N_{0} / 2$ remains even. Its $u \rightarrow 0$ limit that is given by (A.1)-(A.4) has also been checked for states of various lengths $L, N_{0}=2,6,10$ and even $n$ such that $(n+1) N_{0} / 2$ is odd.

\section{B Calculations with the twisted Yangian $Y^{+}(3)$}

In this section we investigate the embeddings $V(s) \subseteq L(s, s, 0)$ and $V(s) \subseteq L(s, s, 1 / 2) \otimes$ $V(1 / 2)$ for the twisted Yangian $Y^{+}(3)$.

\section{B.1 Odd $k=2 s+1$}

$s=1$. For $s=1$, the $\mathfrak{g l}_{3}$ module $L(1,1,0)$ has the same dimension as $V(1)$ and the two have the same $Y^{+}(3)$ highest weights therefore $L(1,1,0) \cong V(1)$ as $Y^{+}(3)$ representation.

$\boldsymbol{s}=\mathbf{2}$. For $s=2$, the states of $L(2,2,0)$ are shown in table 2 where

$$
\begin{aligned}
& |2,1,1\rangle=E_{1,0}|2,2,0\rangle \\
& |2,0,2\rangle=E_{1,0}^{2}|2,2,0\rangle \\
& |1,2,1\rangle=E_{0,-1} E_{1,0}|2,2,0\rangle, \\
& |1,1,2\rangle=E_{0,-1} E_{1,0}^{2}|2,2,0\rangle, \\
& |0,2,2\rangle=E_{0,-1}^{2} E_{1,0}^{2}|2,2,0\rangle .
\end{aligned}
$$

We can see that the $V(2)$ subspace has to be built from the vectors $|2,2,0\rangle$, $|2,1,1\rangle,|2,0,2\rangle+a|1,2,1\rangle,|1,1,2\rangle,|0,2,2\rangle$. a can be calculated from the fact that 


\begin{tabular}{|rll|}
\hline$S_{z}=3$ & $|3,3,0\rangle$ & \\
2 & $|3,2,1\rangle$ & \\
1 & $|3,1,2\rangle$ & $|2,3,1\rangle$ \\
0 & $|3,0,3\rangle$ & $|2,2,2\rangle$ \\
-1 & $|2,1,3\rangle$ & $|1,3,2\rangle$ \\
-2 & $|1,2,3\rangle$ & \\
-3 & $|0,3,3\rangle$ & \\
\hline
\end{tabular}

Table 3. The states of $L(3,3,0)$.

$$
\begin{aligned}
& s_{1,-1}(u) \cdot|2,2,0\rangle \in V(2): \\
& \begin{aligned}
s_{1,-1}(u) \cdot|2,2,0\rangle= & t_{1,1}(u) t_{1,-1}(-u) \cdot|2,2,0\rangle+t_{1,0}(u) t_{1,0}(-u) \cdot|2,2,0\rangle+ \\
& +t_{1,-1}(u) t_{1,1}(-u) \cdot|2,2,0\rangle= \\
= & -u^{-1}\left(1+u^{-1}\right) E_{1,-1}|2,2,0\rangle-u^{-2}|2,0,2\rangle+u^{-1} E_{1,-1}|2,2,0\rangle= \\
= & u^{-2}(|1,2,1\rangle-|2,0,2\rangle),
\end{aligned}
\end{aligned}
$$

therefore $a=-1$.

Let us define the sub-quotient $L(2,2,0) \backslash V(2)$ by $W$. The $W$ is a one-dimensional highest weight representation of $Y^{+}(3)$. Let us calculate the highest weights.

$$
\begin{aligned}
& s_{11}(u) \cdot|2,0,2\rangle=t_{1,1}(u) t_{-1,-1}(-u) \cdot|2,0,2\rangle=\left(1-4 u^{-2}\right)|2,2,0\rangle, \\
& s_{00}(u) \cdot|1,2,1\rangle=t_{0,0}(u) t_{0,0}(-u) \cdot|1,2,1\rangle=\left(1-4 u^{-2}\right)|2,2,0\rangle .
\end{aligned}
$$

Therefore

$$
\begin{aligned}
& \mu_{1}(u)=\left(1-4 u^{-2}\right), \\
& \mu_{0}(u)=\left(1-4 u^{-2}\right),
\end{aligned}
$$

which are the highest weights of the one-dimensional irrep $L(2,2,2)$. Hence, for $L(2,2,0) \cong$ $V(2) \oplus L(2,2,2)$ the action of $Y^{+}(3)$ reads

$$
s_{i j}(u) \cdot v=\mathcal{L}_{i a}^{(2,2,0)}(u) \mathcal{L}_{-j,-a}^{(2,2,0)}(-u) v=\left(\begin{array}{cc}
\psi_{i j}^{(2)}(u) & X \\
0 & \mathcal{L}_{i a}^{(2,2,2)}(u) \mathcal{L}_{-j,-a}^{(2,2,2)}(-u)
\end{array}\right)\left(\begin{array}{l}
w_{1} \\
w_{2}
\end{array}\right),
$$

where $v \in L(2,2,0), w_{1} \in V(2)$ and $w_{2} \in L(2,2,2)$.

$\boldsymbol{s}=3$. Let us continue with $s=3$. Table 3 shows the states of $L(3,3,0)$. We can see that the $V(3)$ subspace has to be built from the vectors $|3,3,0\rangle,|3,2,1\rangle,|3,1,2\rangle+a_{1}|2,3,1\rangle$, $|3,0,3\rangle+a_{2}|2,2,2\rangle,|2,1,3\rangle+a_{3}|1,3,2\rangle,|1,2,3\rangle,|0,3,3\rangle . a_{1}$ can be calculated from the fact that $s_{1,-1}(u) \cdot|3,3,0\rangle \in V(3)$ :

$$
\begin{aligned}
s_{1,-1}(u) \cdot|3,3,0\rangle= & t_{1,1}(u) t_{1,-1}(-u) \cdot|3,3,0\rangle+t_{1,0}(u) t_{1,0}(-u) \cdot|3,3,0\rangle+ \\
& +t_{1,-1}(u) t_{1,1}(-u) \cdot|3,3,0\rangle= \\
= & -u^{-1}\left(1+u^{-1}\right) E_{1,-1}|3,3,0\rangle-u^{-2}|3,1,2\rangle+u^{-1} E_{1,-1}|3,3,0\rangle= \\
= & u^{-2}(|2,3,1\rangle-|3,1,2\rangle),
\end{aligned}
$$

therefore $a_{1}=-1$. 


\begin{tabular}{rr|}
\hline$S_{z}=1$ & $|3,3,2\rangle$ \\
0 & $|3,2,3\rangle$ \\
-1 & $|2,3,3\rangle$ \\
\hline
\end{tabular}

Table 4. The states of $L(3,3,2)$.

Let us define the sub-quotient $L(3,3,0) \backslash V(3)$ by $W$. $W$ is a three-dimensional highest weight representation of $Y^{+}(3)$. Let us calculate the highest weights.

$$
\begin{aligned}
& s_{11}(u) \cdot|3,1,2\rangle=t_{1,1}(u) t_{-1,-1}(-u) \cdot|3,1,2\rangle=\left(1+2 u^{-1}\right)\left(1-3 u^{-1}\right)|3,1,2\rangle, \\
& s_{00}(u) \cdot|2,3,1\rangle=t_{0,0}(u) t_{0,0}(-u) \cdot|2,3,1\rangle=\left(1-9 u^{-2}\right)|2,3,1\rangle .
\end{aligned}
$$

Therefore

$$
\begin{aligned}
& \mu_{1}(u)=\left(1+2 u^{-1}\right)\left(1-3 u^{-1}\right), \\
& \mu_{0}(u)=\left(1-9 u^{-2}\right) .
\end{aligned}
$$

We can see that these are the highest weights of $L(3,3,2) . L(3,3,2)$ is an $Y^{+}(3)$ irrep (see table 4) i.e. for $L(3,3,0) \cong V(3) \oplus L(3,3,2)$ the action of $Y^{+}(3)$ reads

$$
s_{i j}(u) \cdot v=\mathcal{L}_{i a}^{(3,3,0)}(u) \mathcal{L}_{-j,-a}^{(3,3,0)}(-u) v=\left(\begin{array}{cc}
\psi_{i j}^{(3)}(u) & X \\
0 & \mathcal{L}_{i a}^{(3,3,2)}(u) \mathcal{L}_{-j,-a}^{(3,3,2)}(-u)
\end{array}\right)\left(\begin{array}{l}
w_{1} \\
w_{2}
\end{array}\right),
$$

where $v \in L(3,3,0), w_{1} \in V(3)$ and $w_{2} \in L(3,3,2)$.

$s=4$. Similarly, for $L(4,4,0)$ we can define the sub-quotient $W=L(4,4,0) \backslash V(4)$. The highest weights of it are the following

$$
\begin{aligned}
& \mu_{1}(u)=\left(1+2 u^{-1}\right)\left(1-4 u^{-1}\right), \\
& \mu_{0}(u)=\left(1-16 u^{-2}\right) .
\end{aligned}
$$

These are also the highest weights of $L(4,4,2)$. We can show that this is an $Y^{+}(3)$ irrep. The states are shown in table 5 . We only have to show that the subspace $s_{i j}(u) \cdot|4,4,2\rangle$ contains the two-dimensional subspace $\operatorname{span}(\{|4,2,4\rangle,|3,4,3\rangle\})$. This can be done as follows

$$
\begin{aligned}
s_{1,0}(u) \cdot|4,3,3\rangle= & t_{1,1}(u) t_{0,-1}(-u) \cdot|4,3,3\rangle+t_{1,0}(u) t_{0,0}(-u) \cdot|4,3,3\rangle+ \\
& +t_{1,-1}(u) t_{0,1}(-u) \cdot|4,3,3\rangle= \\
= & -u^{-1}\left(1+3 u^{-1}\right)|3,4,3\rangle+u^{-1}\left(1-3 u^{-1}\right)|4,2,4\rangle-2 u^{-2} \Lambda_{1,-1}|4,4,2\rangle= \\
= & u^{-1}(|4,2,4\rangle-|3,4,3\rangle)-u^{-2}(3|4,2,4\rangle+|3,4,3\rangle) .
\end{aligned}
$$

Since $L(4,4,2)$ is an irrep with the same highest weights as $W$ therefore $W \cong L(4,4,2)$ as $Y^{+}(3)$ representations i.e. for the vector space decomposition $L(4,4,0) \cong V(4) \oplus L(4,4,2)$ the action of $Y^{+}(3)$ reads

$$
s_{i j}(u) \cdot v=\mathcal{L}_{i a}^{(4,4,0)}(u) \mathcal{L}_{-j,-a}^{(4,4,0)}(-u) v=\left(\begin{array}{cc}
\psi_{i j}^{(4)}(u) & X \\
0 & \mathcal{L}_{i a}^{(4,4,2)}(u) \mathcal{L}_{-j,-a}^{(4,4,2)}(-u)
\end{array}\right)\left(\begin{array}{l}
w_{1} \\
w_{2}
\end{array}\right),
$$

where $v \in L(4,4,0), w_{1} \in V(4)$ and $w_{2} \in L(4,4,2)$. 


$$
\begin{array}{rll}
S_{z}=2 & |4,4,2\rangle & \\
1 & |4,3,3\rangle & \\
0 & |4,2,4\rangle & |3,4,3\rangle \\
-1 & |3,1,4\rangle & \\
-2 & |2,4,4\rangle &
\end{array}
$$

Table 5. The states of $L(4,4,2)$.

$$
\begin{aligned}
& S_{z}=\frac{3}{2} \quad\left|\frac{3}{2}, \frac{3}{2}, \frac{1}{2}\right\rangle\left|+\frac{1}{2}\right\rangle \\
& \frac{1}{2} \quad\left|\frac{3}{2}, \frac{1}{2}, \frac{3}{2}\right\rangle\left|+\frac{1}{2}\right\rangle \quad\left|\frac{3}{2}, \frac{3}{2}, \frac{1}{2}\right\rangle\left|-\frac{1}{2}\right\rangle \\
& -\frac{1}{2} \quad\left|\frac{1}{2}, \frac{3}{2}, \frac{3}{2}\right\rangle\left|+\frac{1}{2}\right\rangle \quad\left|\frac{3}{2}, \frac{1}{2}, \frac{3}{2}\right\rangle\left|-\frac{1}{2}\right\rangle \\
& -\frac{3}{2} \quad\left|\frac{1}{2}, \frac{3}{2}, \frac{3}{2}\right\rangle\left|-\frac{1}{2}\right\rangle
\end{aligned}
$$

Table 6. The states of $L(3 / 2,3 / 2,1 / 2) \otimes V(1 / 2)$.

General $s$. This can be generalized to $L(s, s, 0) \cong V(s) \oplus L(s, s, 2)$ with the action

$$
s_{i j}(u) \cdot v=\mathcal{L}_{i a}^{(s, s, 0)}(u) \mathcal{L}_{-j,-a}^{(s, s, 0)}(-u) v=\left(\begin{array}{cc}
\psi_{i j}^{(s)}(u) & X \\
0 & \mathcal{L}_{i a}^{(s, s, 2)}(u) \mathcal{L}_{-j,-a}^{(s, s, 2)}(-u)
\end{array}\right)\left(\begin{array}{l}
w_{1} \\
w_{2}
\end{array}\right)
$$

where $v \in L(s, s, 0), w_{1} \in V(s), w_{2} \in L(s, s, 2)$ and $s \in \mathbb{Z}_{>1}$ where we used conjecture 1 .

\section{B.2 Even $k=2 s+1$}

$s=\mathbf{3} / \mathbf{2}$. Let us start with $s=3 / 2$. The states are shown in table 6 . Let us define the sub-quotient $L(3 / 2,3 / 2,1 / 2) \otimes V(1 / 2) \backslash V(3 / 2)$ by $W$. $W$ is a two-dimensional highest weight representation of $Y^{+}(3)$. Let us calculate the highest weights:

$$
\begin{aligned}
s_{11}(u) \cdot\left|\frac{3}{2}, \frac{1}{2}, \frac{3}{2}\right\rangle\left|+\frac{1}{2}\right\rangle & =t_{1,1}(u) t_{-1,-1}(-u) \cdot\left|\frac{3}{2}, \frac{1}{2}, \frac{3}{2}\right\rangle \psi_{1,1}(u)\left|+\frac{1}{2}\right\rangle= \\
& =\left(1-\frac{9}{4} u^{-2}\right)\left(1-\frac{1}{2} u^{-1}\right)\left|\frac{3}{2}, \frac{1}{2}, \frac{3}{2}\right\rangle\left|+\frac{1}{2}\right\rangle, \\
s_{00}(u) \cdot\left|\frac{3}{2}, \frac{3}{2}, \frac{1}{2}\right\rangle\left|-\frac{1}{2}\right\rangle & =t_{0,0}(u) t_{0,0}(-u) \cdot\left|\frac{3}{2}, \frac{3}{2}, \frac{1}{2}\right\rangle \psi_{0,0}(u)\left|-\frac{1}{2}\right\rangle= \\
& =\left(1-\frac{9}{4} u^{-2}\right)\left(1-\frac{1}{4} u^{-2}\right)\left|\frac{3}{2}, \frac{3}{2}, \frac{1}{2}\right\rangle\left|-\frac{1}{2}\right\rangle .
\end{aligned}
$$

Therefore the highest weights are

$$
\begin{aligned}
& \mu_{1}(u)=\left(1-\frac{9}{4} u^{-2}\right)\left(1-\frac{1}{2} u^{-1}\right), \\
& \mu_{0}(u)=\left(1-\frac{9}{4} u^{-2}\right)\left(1-\frac{1}{4} u^{-2}\right),
\end{aligned}
$$




\begin{tabular}{|c|c|c|c|}
\hline $\begin{array}{r}S_{z}=\frac{5}{2} \\
\frac{3}{2} \\
\frac{1}{2} \\
-\frac{1}{2} \\
-\frac{3}{2} \\
-\frac{5}{2}\end{array}$ & $\begin{array}{l}\left|\frac{5}{2}, \frac{5}{2}, \frac{1}{2}\right\rangle\left|+\frac{1}{2}\right\rangle \\
\left|\frac{5}{2}, \frac{3}{2}, \frac{3}{2}\right\rangle\left|+\frac{1}{2}\right\rangle \\
\left|\frac{5}{2}, \frac{1}{2}, \frac{5}{2}\right\rangle\left|+\frac{1}{2}\right\rangle \\
\left|\frac{3}{2}, \frac{3}{2}, \frac{5}{2}\right\rangle\left|+\frac{1}{2}\right\rangle \\
\left|\frac{1}{2}, \frac{5}{2}, \frac{5}{2}\right\rangle\left|+\frac{1}{2}\right\rangle \\
\left|\frac{1}{2}, \frac{5}{2}, \frac{5}{2}\right\rangle\left|-\frac{1}{2}\right\rangle\end{array}$ & $\begin{array}{l}\left|\frac{5}{2}, \frac{5}{2}, \frac{1}{2}\right\rangle\left|-\frac{1}{2}\right\rangle \\
\left|\frac{5}{2}, \frac{3}{2}, \frac{3}{2}\right\rangle\left|-\frac{1}{2}\right\rangle \\
\left|\frac{5}{2}, \frac{1}{2}, \frac{5}{2}\right\rangle\left|-\frac{1}{2}\right\rangle \\
\left|\frac{3}{2}, \frac{3}{2}, \frac{5}{2}\right\rangle\left|-\frac{1}{2}\right\rangle\end{array}$ & $\begin{array}{l}\left|\frac{3}{2}, \frac{5}{2}, \frac{3}{2}\right\rangle\left|+\frac{1}{2}\right\rangle \\
\left|\frac{3}{2}, \frac{5}{2}, \frac{3}{2}\right\rangle\left|-\frac{1}{2}\right\rangle\end{array}$ \\
\hline
\end{tabular}

Table 7. The states of $L(5 / 2,5 / 2,1 / 2) \otimes V(1 / 2)$.

i.e. for $(L(3 / 2,3 / 2,1 / 2) \otimes V(1 / 2)) \cong V(3 / 2) \oplus(L(3 / 2,3 / 2,3 / 2) \otimes V(1 / 2))$ the $Y^{+}(3)$ action is

$$
\begin{aligned}
& s_{i j}(u) \cdot\left(v_{1} \otimes v_{2}\right)=\mathcal{L}_{i a}^{(3 / 2,3 / 2,1 / 2)}(u) \mathcal{L}_{-j,-b}^{(3 / 2,3 / 2,1 / 2)}(-u) v_{1} \otimes \psi_{a, b}^{(1 / 2)}(u) v_{2}= \\
& =\left(\begin{array}{cc}
\left(1-\frac{1}{4} u^{-2}\right) \psi_{i, j}^{(3 / 2)}(u) & X \\
0 & \mathcal{L}_{i a}^{(3 / 2,3 / 2,3 / 2)}(u) \mathcal{L}_{-j,-b}^{(3 / 2,3 / 2,3 / 2)}(-u) \otimes \psi_{a, b}^{(1 / 2)}(u)
\end{array}\right)\left(\begin{array}{c}
w_{1} \\
w_{2} \otimes w_{3}
\end{array}\right),
\end{aligned}
$$

for all $v_{1} \in L(3 / 2,3 / 2,1 / 2), v_{2}, w_{3} \in V(1 / 2), w_{1} \in V(3 / 2)$ and $w_{2} \in L(3 / 2,3 / 2,3 / 2)$.

$s=\mathbf{5} / \mathbf{2}$. Let us continue with $s=5 / 2$. Table 7 shows the states of $L(5 / 2,5 / 2,1 / 2) \otimes$ $V(1 / 2)$. Let us define the sub-quotient $L(5 / 2,5 / 2,1 / 2) \otimes V(1 / 2) / V(5 / 2)$ by $W$. $W$ is a six-dimensional highest weight representation of $Y^{+}(3)$. We calculate the highest weights:

$$
\begin{aligned}
s_{11}(u) \cdot\left|\frac{5}{2}, \frac{3}{2}, \frac{3}{2}\right\rangle\left|+\frac{1}{2}\right\rangle & =t_{1,1}(u) t_{-1,-1}(-u) \cdot\left|\frac{5}{2}, \frac{3}{2}, \frac{3}{2}\right\rangle \psi_{1,1}(u)\left|+\frac{1}{2}\right\rangle= \\
& =\left(1+\frac{3}{2} u^{-1}\right)\left(1-\frac{5}{2} u^{-1}\right)\left(1-\frac{1}{2} u^{-1}\right)\left|\frac{5}{2}, \frac{3}{2}, \frac{3}{2}\right\rangle\left|+\frac{1}{2}\right\rangle, \\
s_{00}(u) \cdot\left|\frac{5}{2}, \frac{5}{2}, \frac{1}{2}\right\rangle\left|-\frac{1}{2}\right\rangle & =t_{0,0}(u) t_{0,0}(-u) \cdot\left|\frac{5}{2}, \frac{5}{2}, \frac{1}{2}\right\rangle \psi_{0,0}(u)\left|-\frac{1}{2}\right\rangle= \\
& =\left(1-\frac{25}{4} u^{-2}\right)\left(1-\frac{1}{4} u^{-2}\right)\left|\frac{3}{2}, \frac{3}{2}, \frac{1}{2}\right\rangle\left|-\frac{1}{2}\right\rangle .
\end{aligned}
$$

Therefore the highest weights are

$$
\begin{aligned}
& \mu_{1}(u)=\left(1+\frac{3}{2} u^{-1}\right)\left(1-\frac{5}{2} u^{-1}\right)\left(1-\frac{1}{2} u^{-1}\right) \\
& \mu_{0}(u)=\left(1-\frac{25}{4} u^{-2}\right)\left(1-\frac{1}{4} u^{-2}\right)
\end{aligned}
$$

We can see that these are the highest weights of $L(5 / 2,5 / 2,3 / 2) \otimes V(1 / 2)$. We can show that this is an $Y^{+}(3)$ irrep. The states are shown in table 8. 


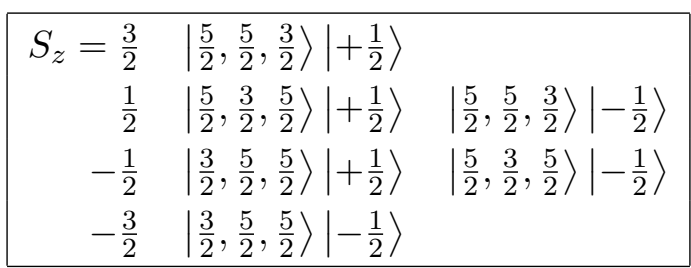

Table 8. The states of $L(5 / 2,5 / 2,3 / 2) \otimes V(1 / 2)$.

\section{From}

$$
\begin{aligned}
s_{1,0}(u) \cdot\left|\frac{5}{2}, \frac{5}{2}, \frac{3}{2}\right\rangle\left|+\frac{1}{2}\right\rangle= & t_{1,0}(u) t_{0,0}(-u) \cdot\left|\frac{5}{2}, \frac{5}{2}, \frac{3}{2}\right\rangle \psi_{0,0}(u)\left|+\frac{1}{2}\right\rangle+ \\
& +t_{1,1}(u) t_{0,0}(-u) \cdot\left|\frac{5}{2}, \frac{5}{2}, \frac{3}{2}\right\rangle \psi_{1,0}(u)\left|+\frac{1}{2}\right\rangle= \\
= & u^{-1}\left(1-\frac{5}{2} u^{-1}\right)\left(1-\frac{1}{4} u^{-2}\right)\left|\frac{5}{2}, \frac{3}{2}, \frac{5}{2}\right\rangle\left|+\frac{1}{2}\right\rangle+ \\
& +\frac{2 i}{\sqrt{2}} u^{-1}\left(1+\frac{3}{2} u^{-1}\right)\left(1-\frac{5}{2} u^{-1}\right)\left(1-\frac{1}{4} u^{-1}\right)\left|\frac{5}{2}, \frac{3}{2}, \frac{5}{2}\right\rangle\left|+\frac{1}{2}\right\rangle,
\end{aligned}
$$

we can see that $L(5 / 2,5 / 2,3 / 2) \otimes V(1 / 2)$ is irreducible, i.e. for $(L(5 / 2,5 / 2,1 / 2) \otimes V(1 / 2)) \cong$ $V(5 / 2) \oplus(L(5 / 2,5 / 2,3 / 2) \otimes V(1 / 2))$ the action can be written as

$$
\begin{aligned}
& s_{i j}(u) \cdot\left(v_{1} \otimes v_{2}\right)=\mathcal{L}_{i a}^{(5 / 2,5 / 2,1 / 2)}(u) \mathcal{L}_{-j,-b}^{(5 / 2,5 / 2,1 / 2)}(-u) v_{1} \otimes \psi_{a, b}^{(1 / 2)}(u) v_{2}= \\
& =\left(\begin{array}{cc}
\left(1-\frac{1}{4} u^{-2}\right) \psi_{i, j}^{(5 / 2)}(u) & X \\
0 & \mathcal{L}_{i a}^{(5 / 2,5 / 2,3 / 2)}(u) \mathcal{L}_{-j,-b}^{(5 / 2,5 / 2,3 / 2)}(-u) \otimes \psi_{a, b}^{(1 / 2)}(u)
\end{array}\right)\left(\begin{array}{c}
w_{1} \\
w_{2} \otimes w_{3}
\end{array}\right),
\end{aligned}
$$

for all $v_{1} \in L(5 / 2,5 / 2,1 / 2), v_{2}, w_{3} \in V(1 / 2), w_{1} \in V(5 / 2)$ and $w_{2} \in L(5 / 2,5 / 2,3 / 2)$.

General $s$. This can be generalized to $(L(s, s, 1 / 2) \otimes V(1 / 2)) \cong V(s) \oplus(L(s, s, 3 / 2) \otimes V(1 / 2))$ with the action

$$
\begin{aligned}
& s_{i j}(u) \cdot\left(v_{1} \otimes v_{2}\right)=\mathcal{L}_{i a}^{(s, s, 1 / 2)}(u) \mathcal{L}_{-j,-b}^{(s, s, 1 / 2)}(-u) v_{1} \otimes \psi_{a, b}^{(1 / 2)}(u) v_{2}= \\
& \quad=\left(\begin{array}{cc}
\left(1-\frac{1}{4} u^{-2}\right) \psi_{i, j}^{(s)}(u) & X \\
0 & \mathcal{L}_{i a}^{(s, s, 3 / 2)}(u) \mathcal{L}_{-j,-b}^{(s, s, 3 / 2)}(-u) \otimes \psi_{a, b}^{(1 / 2)}(u)
\end{array}\right)\left(\begin{array}{c}
w_{1} \\
w_{2} \otimes w_{3}
\end{array}\right),
\end{aligned}
$$

for all $v_{1} \in L(s / 2, s / 2,1 / 2), v_{2}, w_{3} \in V(1 / 2), w_{1} \in V(s / 2), w_{2} \in L(s / 2, s / 2,3 / 2)$ and $s \in \mathbb{Z}_{+}+\frac{1}{2}$ where we used conjecture 2 . 


\section{Calculations with the twisted Yangian $X\left(\mathfrak{s o}_{6}, \mathfrak{s o}_{5}\right)$}

From the explicit forms

$$
\begin{aligned}
s_{3,3}(u) & =\tilde{g}_{1}(u) \tilde{G}_{1} \tilde{G}_{-1}+\tilde{g}_{2}(u)\left[\tilde{G}_{1}, \tilde{G}_{-1}\right]+\tilde{f}(u), \\
s_{2,2}(u) & =\tilde{g}_{1}(u) \tilde{G}_{2} \tilde{G}_{-2}+\tilde{g}_{2}(u)\left[\tilde{G}_{2}, \tilde{G}_{-2}\right]+\tilde{f}(u), \\
s_{1,1}(u) & =\frac{1}{2}\left(\tilde{g}_{1}(u) \tilde{G}_{0}^{2}+\tilde{f}(u)+\tilde{h}(u)\right), \\
s_{1,-1}(u) & =\frac{1}{2}\left(\tilde{g}_{1}(u) \tilde{G}_{0}^{2}+\tilde{f}(u)-\tilde{h}(u)\right)=s_{-1,1}(u),
\end{aligned}
$$

we can calculate the weights $\mu_{3}(u), \mu_{2}(u), \mu_{1}(u)$ and $\mu^{(+)}(u)=\mu^{(-)}(u)$.

Let us define the $\mathfrak{s o}_{5}$ generators

$$
F_{i j}=\frac{1}{4}\left[G_{i}, G_{-j}\right]
$$

for which

$$
\left[F_{i j}, \tilde{G}_{k}\right]=\delta_{j k} \tilde{G}_{i}-\delta_{-i, k} \tilde{G}_{-j}
$$

The $F_{i j}$ s form a h.w. representation of $\mathfrak{s o}_{5}$ i.e. there exists a vector $v$ for which $\left(\lambda_{1}, \lambda_{2}\right)=$ $\left(-\frac{n}{2},-\frac{n}{2}\right)$. From (C.6) we can see that $\tilde{G}_{1}$ and $\tilde{G}_{2}$ increase the weights and $\tilde{G}_{-1}$ and $\tilde{G}_{-2}$ decrease the weights, therefore

$$
G_{-1} \cdot v=G_{-2} \cdot v=0 .
$$

Using this we can calculate $\mu_{3}$ and $\mu_{2}$ as

$$
\begin{aligned}
& s_{3,3}(u) \cdot v=\left(\tilde{f}(u)+4 \tilde{g}_{2}(u) F_{1,1}\right) \cdot v=\left(\tilde{f}(u)-2 n \tilde{g}_{2}(u)\right) v, \\
& s_{2,2}(u) \cdot v=\left(\tilde{f}(u)+4 \tilde{g}_{2}(u) F_{2,2}\right) \cdot v=\left(\tilde{f}(u)-2 n \tilde{g}_{2}(u)\right) v .
\end{aligned}
$$

For $\mu_{1}$ and $\mu^{(+)}$we have to calculate how $\tilde{G}_{0}^{2}$ act on $v$. This can be done using (3.14)

$$
\begin{aligned}
\tilde{G}_{0}^{2} \cdot v & =C v-\left(\tilde{G}_{1} \tilde{G}_{-1}+\tilde{G}_{-1} \tilde{G}_{1}+\tilde{G}_{2} \tilde{G}_{-2}+\tilde{G}_{-2} \tilde{G}_{2}\right) \cdot v= \\
& =C v+4\left(F_{11}+F_{22}\right) \cdot v=(C-4 n) v=n^{2} v .
\end{aligned}
$$

Using this we obtain that

$$
\begin{aligned}
& s_{1,1}(u) \cdot v=\frac{1}{2}\left(n^{2} \tilde{g}_{1}(u)+\tilde{f}(u)+\tilde{h}(u)\right) \cdot v, \\
& s_{2,2}(u) \cdot v=\frac{1}{2}\left(n^{2} \tilde{g}_{1}(u)+\tilde{f}(u)-\tilde{h}(u)\right) \cdot v,
\end{aligned}
$$

therefore

$$
\begin{gathered}
\mu_{3}(u)=\mu_{2}(u)=\left(1-u^{-1}\right)\left(1-\frac{n}{2} u^{-1}\right)^{2}, \\
\mu_{1}(u)=-u^{-1}\left(1-u^{-1}\right)\left(1-\frac{n}{2} u^{-1}\right)^{2}, \\
\mu^{(+)}(u)=\mu^{(-)}(u)=\left(1-u^{-2}\right)\left(1-\frac{n^{2}}{4} u^{-2}\right) .
\end{gathered}
$$


Now let us try to obtain this S-matrix in the form

$$
S^{D}(u)=T^{\mathfrak{s o}_{6}}(u) K(u)\left(T^{\mathfrak{s o}_{6}}\right)^{t}(-u),
$$

using some $\mathfrak{g l}_{4}$ module $L\left(\lambda_{1}, \lambda_{2}, \lambda_{3}, \lambda_{4}\right)$. The $\mathfrak{s o}_{5}$ is embedded in $\mathfrak{g l}_{4}$ and $X\left(\mathfrak{s o}_{6}, \mathfrak{s o}_{5}\right)$. Our S-matrix and $L\left(\lambda_{1}, \lambda_{2}, \lambda_{3}, \lambda_{4}\right)$ have highest weights $\left(-\frac{n}{2},-\frac{n}{2}\right)$ and $\left(-\frac{\lambda_{1}+\lambda_{2}-\lambda_{3}-\lambda_{4}}{2}\right.$, $\left.-\frac{\lambda_{1}-\lambda_{2}+\lambda_{3}-\lambda_{4}}{2}\right)$ as $\mathfrak{s o}_{5}$ modules. Therefore the $\mathfrak{g l}_{4}$ highest weights have to be $(n+c, a, a, c)$. Matching the dimensions of the representation, only two options remain:

1. $(n+c, n+c, n+c, c)$ or

2. $(n+c, c, c, c)$.

Let us calculate the $X\left(\mathfrak{s o}_{6}, \mathfrak{s o}_{5}\right)$ highest weights of the first one.

$$
\begin{aligned}
s_{3,3}^{D}(u) \cdot v & =\frac{u}{u+1} t_{3,3}^{\mathfrak{s o}_{6}}(u) t_{-3,-3}^{\mathfrak{s o}_{6}}(u) \cdot v= \\
& =\frac{u}{u+1} t_{33}^{\mathfrak{g l}_{4}}(u) t_{44}^{\mathfrak{g l}_{4}}(u-1) t_{11}^{\mathfrak{g l}_{4}}(-u) t_{22}^{\mathfrak{g l}_{4}}(-u-1) \cdot v= \\
& =\frac{(u+n+c)(u+c-1)(u-n-c)(u-n-c+1)}{u(u+1)^{2}(u-1)} \cdot v,
\end{aligned}
$$

where we used that $t_{i, j}^{\mathfrak{s o}_{6}}(u) \cdot v=t_{i, j}^{\mathfrak{g l}_{4}}(u) \cdot v=0$ for $i<j$. In an analogous way we can calculate the other highest weights and the result is

$$
\begin{aligned}
\mu_{3}^{D}(u) & =\mu_{2}^{D}(u)=\frac{\left(u^{2}-(n+c)^{2}\right)(u+c-1)(u-n-c+1)}{u(u+1)^{2}(u-1)}, \\
\mu_{1}^{D}(u) & =-\frac{\left(u^{2}-(n+c)^{2}\right)(u+c-1)(u-n-c+1)}{u^{2}(u+1)^{2}(u-1)}, \\
\mu^{D(+)}(u) & =\frac{\left(u^{2}-(n+c)^{2}\right)\left(u^{2}-(n+c-1)^{2}\right)}{u^{2}\left(u^{2}-1\right)}, \\
\mu^{D(-)}(u) & =\frac{\left(u^{2}-(n+c)^{2}\right)\left(u^{2}-(c-1)^{2}\right)}{u^{2}\left(u^{2}-1\right)} .
\end{aligned}
$$

The equation (C.15) implies that $\mu^{D(+)}(u)$ has to be equal to $\mu^{D(-)}(u)$ therefore

$$
c=1-\frac{n}{2} \text {. }
$$

After substitution

$$
\begin{gathered}
\mu_{3}^{D}(u)=\mu_{2}^{D}(u)=\frac{\left(1-\left(\frac{n}{2}+1\right)^{2} u^{-2}\right)\left(1-\frac{n}{2} u^{-1}\right)^{2}}{\left(1+u^{-1}\right)^{2}\left(1-u^{-1}\right)}=\frac{\left(1-\left(\frac{n}{2}+1\right)^{2} u^{-2}\right)}{\left(1-u^{-2}\right)^{2}} \mu_{3}(u), \\
\mu_{1}^{D}(u)=-u^{-1} \frac{\left(1-\left(\frac{n}{2}+1\right)^{2} u^{-2}\right)\left(1-\frac{n}{2} u^{-1}\right)^{2}}{\left(1+u^{-1}\right)^{2}\left(1-u^{-1}\right)}=\frac{\left(1-\left(\frac{n}{2}+1\right)^{2} u^{-2}\right)}{\left(1-u^{-2}\right)^{2}} \mu_{1}(u), \\
\mu^{D(+)}(u)=\mu^{D(-)}(u)=\frac{\left(1-\left(\frac{n}{2}+1\right)^{2} u^{-2}\right)\left(1-\frac{n^{2}}{4} u^{-2}\right)}{\left(1-u^{-2}\right)}=\frac{\left(1-\left(\frac{n}{2}+1\right)^{2} u^{-2}\right)}{\left(1-u^{-2}\right)^{2}} \mu^{(+)}(u) .
\end{gathered}
$$


Therefore we obtain that

$$
S(u)=\frac{\left(1-u^{-2}\right)^{2}}{\left(1-\left(\frac{n}{2}+1\right)^{2} u^{-2}\right)} T^{\mathfrak{s 0} 6}(u) K(u)\left(T^{\mathfrak{s} 0_{6}}\right)^{t}(-u) .
$$

Open Access. This article is distributed under the terms of the Creative Commons Attribution License (CC-BY 4.0), which permits any use, distribution and reproduction in any medium, provided the original author(s) and source are credited.

\section{References}

[1] J.-S. Caux and F.H.L. Essler, Time evolution of local observables after quenching to an integrable model, Phys. Rev. Lett. 110 (2013) 257203 [arXiv:1301.3806] [INSPIRE].

[2] B. Wouters, J. De Nardis, M. Brockmann, D. Fioretto, M. Rigol and J.-S. Caux, Quenching the anisotropic Heisenberg chain: exact solution and generalized Gibbs ensemble predictions, Phys. Rev. Lett. 113 (2014) 117202 [arXiv:1405.0172].

[3] B. Pozsgay, M. Mestyán, M.A. Werner, M. Kormos, G. Zaránd and G. Takács, Correlations after quantum quenches in the XXZ spin chain: failure of the generalized Gibbs ensemble, Phys. Rev. Lett. 113 (2014) 117203 [arXiv:1405.2843].

[4] M. Mestyán, B. Bertini, L. Piroli and P. Calabrese, Exact solution for the quench dynamics of a nested integrable system, J. Stat. Mech. 1708 (2017) 083103 [arXiv:1705.00851] [INSPIRE].

[5] L. Piroli, B. Pozsgay and E. Vernier, What is an integrable quench?, Nucl. Phys. B 925 (2017) 362 [arXiv:1709.04796] [INSPIRE].

[6] L. Piroli, E. Vernier, P. Calabrese and B. Pozsgay, Integrable quenches in nested spin chains I: the exact steady states, J. Stat. Mech. 1906 (2019) 063103 [arXiv:1811.00432] [INSPIRE].

[7] L. Piroli, E. Vernier, P. Calabrese and B. Pozsgay, Integrable quenches in nested spin chains II: fusion of boundary transfer matrices, J. Stat. Mech. 1906 (2019) 063104 [arXiv: 1812.05330] [INSPIRE].

[8] B. Pozsgay, L. Piroli and E. Vernier, Integrable Matrix Product States from boundary integrability, SciPost Phys. 6 (2019) 062 [arXiv: 1812.11094] [INSPIRE].

[9] M. de Leeuw, C. Kristjansen and K. Zarembo, One-point Functions in Defect CFT and Integrability, JHEP 08 (2015) 098 [arXiv: 1506.06958] [INSPIRE].

[10] I. Buhl-Mortensen, M. de Leeuw, C. Kristjansen and K. Zarembo, One-point Functions in AdS/dCFT from Matrix Product States, JHEP 02 (2016) 052 [arXiv: 1512.02532] [INSPIRE].

[11] M. de Leeuw, C. Kristjansen and S. Mori, AdS/dCFT one-point functions of the $\mathrm{SU}(3)$ sector, Phys. Lett. B 763 (2016) 197 [arXiv:1607.03123] [INSPIRE].

[12] M. de Leeuw, C. Kristjansen and G. Linardopoulos, One-point functions of non-protected operators in the SO(5) symmetric D3-D7 dCFT, J. Phys. A 50 (2017) 254001 [arXiv: 1612.06236] [INSPIRE].

[13] M. De Leeuw, C. Kristjansen and G. Linardopoulos, Scalar one-point functions and matrix product states of AdS/dCFT, Phys. Lett. B 781 (2018) 238 [arXiv:1802.01598] [INSPIRE]. 
[14] M. de Leeuw, C. Kristjansen and K.E. Vardinghus, A non-integrable quench from AdS/dCFT, Phys. Lett. B 798 (2019) 134940 [arXiv: 1906.10714] [INSPIRE].

[15] A. Karch and L. Randall, Open and closed string interpretation of SUSY CFT's on branes with boundaries, JHEP 06 (2001) 063 [hep-th/0105132] [INSPIRE].

[16] N.R. Constable, R.C. Myers and O. Tafjord, NonAbelian brane intersections, JHEP 06 (2001) 023 [hep-th/0102080] [INSPIRE].

[17] N.R. Constable, R.C. Myers and O. Tafjord, The Noncommutative bion core, Phys. Rev. D 61 (2000) 106009 [hep-th/9911136] [INSPIRE].

[18] O. DeWolfe, D.Z. Freedman and H. Ooguri, Holography and defect conformal field theories, Phys. Rev. D 66 (2002) 025009 [hep-th/0111135] [InSPIRE].

[19] J. Erdmenger, Z. Guralnik and I. Kirsch, Four-dimensional superconformal theories with interacting boundaries or defects, Phys. Rev. D 66 (2002) 025020 [hep-th/0203020] [INSPIRE].

[20] K. Nagasaki and S. Yamaguchi, Expectation values of chiral primary operators in holographic interface CFT, Phys. Rev. D 86 (2012) 086004 [arXiv:1205.1674] [INSPIRE].

[21] C. Kristjansen, G.W. Semenoff and D. Young, Chiral primary one-point functions in the D3-D7 defect conformal field theory, JHEP 01 (2013) 117 [arXiv: 1210.7015] [INSPIRE].

[22] B. Pozsgay, Overlaps between eigenstates of the XXZ spin-1/2 chain and a class of simple product states, arXiv: 1309.4593.

[23] M. Brockmann, J. De Nardis, B. Wouters and J.-S. Caux, A Gaudin-like determinant for overlaps of Néel and XXZ Bethe states, J. Phys. A 47 (2014) 145003 [arXiv:1401.2877].

[24] M. Brockmann, J. De Nardis, B. Wouters and J.-S. Caux, Néel-XXZ state overlaps: odd particle numbers and Lieb-Liniger scaling limit, J. Phys. A 47 (2014) 345003 [arXiv: 1403.7469].

[25] O. Foda and K. Zarembo, Overlaps of partial Néel states and Bethe states, J. Stat. Mech. 1602 (2016) 023107 [arXiv: 1512.02533] [INSPIRE].

[26] I. Buhl-Mortensen, M. de Leeuw, A.C. Ipsen, C. Kristjansen and M. Wilhelm, Asymptotic One-Point Functions in Gauge-String Duality with Defects, Phys. Rev. Lett. 119 (2017) 261604 [arXiv: 1704.07386] [INSPIRE].

[27] I. Buhl-Mortensen, M. de Leeuw, A.C. Ipsen, C. Kristjansen and M. Wilhelm, One-loop one-point functions in gauge-gravity dualities with defects, Phys. Rev. Lett. 117 (2016) 231603 [arXiv: 1606.01886] [INSPIRE].

[28] I. Buhl-Mortensen, M. de Leeuw, A.C. Ipsen, C. Kristjansen and M. Wilhelm, A Quantum Check of AdS/dCFT, JHEP 01 (2017) 098 [arXiv:1611.04603] [INSPIRE].

[29] J. Castelino, S. Lee and W. Taylor, Longitudinal five-branes as four spheres in matrix theory, Nucl. Phys. B 526 (1998) 334 [hep-th/9712105] [InSPIRE].

[30] A. Gimenez-Grau, C. Kristjansen, M. Volk and M. Wilhelm, A Quantum Framework for AdS/dCFT through Fuzzy Spherical Harmonics on $S^{4}$, arXiv: 1912.02468 [INSPIRE].

[31] D. Gaiotto and E. Witten, Supersymmetric Boundary Conditions in $\mathcal{N}=4$ Super Yang-Mills Theory, J. Statist. Phys. 135 (2009) 789 [arXiv:0804.2902] [inSPIRE].

[32] A.C. Ipsen and K.E. Vardinghus, The dilatation operator for defect conformal $N=4 S Y M$, arXiv: 1909.12181 [INSPIRE]. 
[33] R.C. Myers and M.C. Wapler, Transport Properties of Holographic Defects, JHEP 12 (2008) 115 [arXiv:0811.0480] [INSPIRE].

[34] J.A. Minahan and K. Zarembo, The Bethe ansatz for $N=4$ superYang-Mills, JHEP 03 (2003) 013 [hep-th/0212208] [inSPIRE].

[35] M. Gaudin, La function d'onde de Bethe our les modèles exacts de la mécanique statistique, Commisariat á l'énergie atomique, Paris (1983).

[36] V.E. Korepin, Calculation of norms of Bethe wave functions, Commun. Math. Phys. 86 (1982) 391 [INSPIRE].

[37] M. Kormos and B. Pozsgay, One-Point Functions in Massive Integrable QFT with Boundaries, JHEP 04 (2010) 112 [arXiv: 1002.2783] [INSPIRE].

[38] B. Pozsgay, Overlaps with arbitrary two-site states in the XXZ spin chain, J. Stat. Mech. 1805 (2018) 053103 [arXiv: 1801.03838] [INSPIRE].

[39] B. Basso, F. Coronado, S. Komatsu, H.T. Lam, P. Vieira and D.-l. Zhong, Asymptotic Four Point Functions, JHEP 07 (2019) 082 [arXiv:1701.04462] [INSPIRE].

[40] C. Marboe and D. Volin, Quantum spectral curve as a tool for a perturbative quantum field theory, Nucl. Phys. B 899 (2015) 810 [arXiv:1411.4758] [INSPIRE].

[41] C. Marboe and D. Volin, The full spectrum of AdS5/CFT4 I: Representation theory and one-loop Q-system, J. Phys. A 51 (2018) 165401 [arXiv:1701.03704] [INSPIRE].

[42] C. Marboe, The AdS/CFT spectrum via integrability-based algorithms, Ph.D. thesis, Trinity Coll., Dublin (2017) [INSPIRE].

[43] A. Kuniba, T. Nakanishi and J. Suzuki, T-systems and Y-systems in integrable systems, J. Phys. A 44 (2011) 103001 [arXiv:1010.1344] [InSPIRE].

[44] V. Bazhanov and N. Reshetikhin, Restricted Solid on Solid Models Connected With Simply Based Algebras and Conformal Field Theory, J. Phys. A 23 (1990) 1477 [inSPIRE].

[45] M. Takahashi, M. Shiroishi and A. Klümper, Equivalence of tba and qtm, J. Phys. A 34 (2001) L187 [cond-mat/0102027].

[46] T. Gombor, Nonstandard Bethe Ansatz equations for open $O(N)$ spin chains, Nucl. Phys. B 935 (2018) 310 [arXiv:1712.03753] [INSPIRE].

[47] A. Molev, Finite dimensional irreducible representations of twisted Yangians, J. Math. Phys. 39 (1998) 5559 [q-alg/9711022] [inSPIRE].

[48] D. Arnaudon, A. Molev and E. Ragoucy, On the r-matrix realization of yangians and their representations, Annales Henri Poincaré 7 (2006) 1269.

[49] N. Guay and V. Regelskis, Twisted yangians for symmetric pairs of types b, $c, d$, Math. Z. 284 (2016) 131.

[50] N. Guay, V. Regelskis and C. Wendlandt, Representations of twisted yangians of types $b, c$, d: I, Selecta Math. 23 (2017) 2071.

[51] N. Guay, V. Regelskis and C. Wendlandt, Representations of twisted yangians of types b, $c$, d: Ii, Transform. Groups 24 (2019) 1015.

[52] G.Z. Fehér and B. Pozsgay, Generalized Gibbs Ensemble and string-charge relations in nested Bethe Ansatz, arXiv:1909.04470 [INSPIRE]. 
[53] N. Beisert, The SU(2|2) dynamic S-matrix, Adv. Theor. Math. Phys. 12 (2008) 945 [hep-th/0511082] [INSPIRE].

[54] Y. Jiang, S. Komatsu and E. Vescovi, Structure Constants in $\mathcal{N}=4$ SYM at Finite Coupling as Worldsheet g-Function, arXiv:1906.07733 [INSPIRE].

[55] Y. Jiang, S. Komatsu and E. Vescovi, Exact Three-Point Functions of Determinant Operators in Planar $N=4$ Supersymmetric Yang-Mills Theory, Phys. Rev. Lett. 123 (2019) 191601 [arXiv: 1907.11242] [INSPIRE].

[56] M. de Leeuw, A.C. Ipsen, C. Kristjansen, K.E. Vardinghus and M. Wilhelm, Two-point functions in AdS/dCFT and the boundary conformal bootstrap equations, JHEP 08 (2017) 020 [arXiv: 1705. 03898] [INSPIRE]. 